\title{
A Geoinformatics Approach to Assess Land Use Dynamic and Landscape Fragmentation Due to Opencast Coal Mining in Raniganj Coalfield, India
}

\author{
Amit Sarkar \\ Senior Research Fellow, Department of Geography, University of Calcutta, Kolkata-700019, India \\ Email: iamitsarkar91@gmail.com
}

\begin{abstract}
Land use dynamics and landscape patterns have always been prime themes of global change research (R.H.U. \& Suocheng, D. 2013). Anthropogenic changes in the form of opencast mining make the ecosystem more sensitive and fragmented. Miscellaneous mining aids like dumper, dozer and dragline caused enormous amount of land degradation. Indeed the degraded land covers are fragmented in due course of time. Raniganj coalfield area has a complex land use land cover fragmentation scenario due to opencast mining and associated development activities since 1960 (Das, G. \& Das, R. 2016). Arc GIS and fragstats software is used in order to measure the class level and landscape level fragmentation of different landscapes in Raniganj coalfield and adjoining area since last 40 years. Results denote that most of the landscape classes have become fragmented and isolated. The areas of forest, agricultural land, excavated land and urban tend to be complex in their shape and spatial clustering. The shapes of other land class patches have become less complex, but overall landscape fragmentation has increased during last 25 years. Contrary landscape diversity and heterogeneity have also been increased within only 20 years especially in Sonepur Bazari, Satgram, Sripur and Khottadih area. Therefore it is urgently essential to understand and compute the land use fragmentation process in Raniganj mining area.
\end{abstract}

Keywords: opencast mining, class and landscape level fragmentation, fragstats and Arc GIS

\section{INTRODUCTION}

The researchers strive to interpret land use land cover fragmentation in the focal areas of Raniganj coalfield and its surroundings using remotely sensed data. Opencast coal mining affects the local landscape, adversely causes widespread environmental decay especially land alteration and fragmentation (Maitima, J.M., Mugatha, S.M., Reid, R.S., Gachimbi, L.N., et al. 2009). Therefore preparation and identification of land use land cover fragmentation in temporal manner for any particular area is very crucial nowadays in earth science in order to detect the temporal changes in land use land cover (State, J., Kumar, A. \& Pandey, A.C. 2013). These fragmentation process put forwarded by mining and associated development activities are measured by computing the 11 class level and 2 landscape level fragmentation indices.

\section{RESEARCH AREA}

Raniganj coalfield is located within four districts of West Bengal i.e. Burdwan (71\%), Birbhum (9\%), Bankura (8\%) and Purulia (7\%) shown in figure 2.1. This coalfield is elliptical in shape. East-west extension is about $75 \mathrm{~km}$ and north-south extension is about $35 \mathrm{~km}$. It has an area of about $1530 \mathrm{sq} \mathrm{km}$ and falls within latitudes $23^{\circ} 30^{\prime} \mathrm{N}$ to $23^{\circ} 52^{\prime} \mathrm{N}$ and longitudes $86^{\circ} 38^{\prime} \mathrm{E}$ to $87^{\circ} 23^{\prime} \mathrm{E}$. As on 2016 this coalfield has 17 running OCPs and 21abandoned OCPs within 11 areas (ECL 2015). Mean elevation is $98.45 \mathrm{~m}$ with broad undulation (Sarkar, A., 2016). 


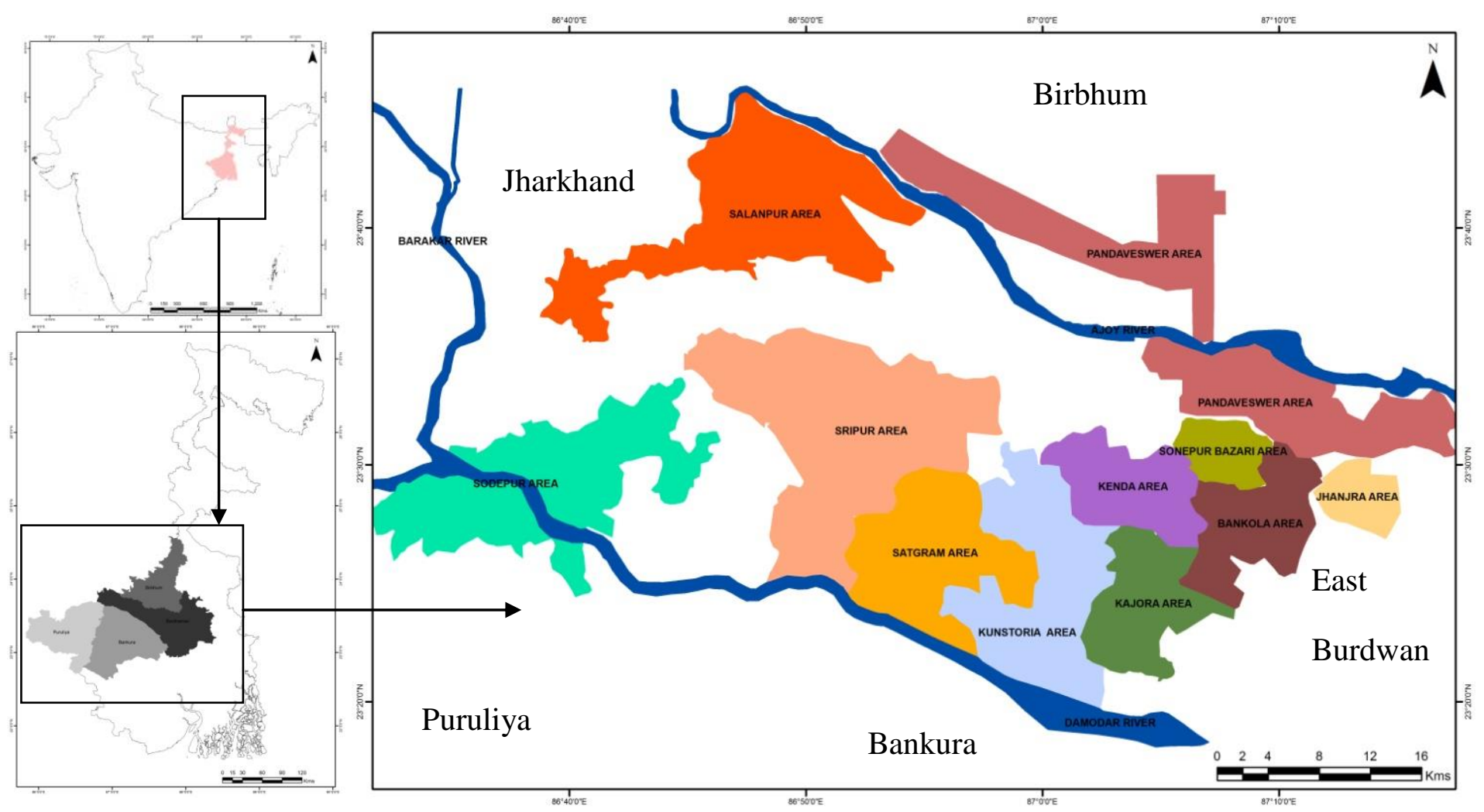

Figure 2.1: Location of Research Area (Source: ECL \& CMPDI)

\section{MATERIALS AND METHODS}

\subsection{Remote Sensing Data Sources}

Five temporal cloud free satellite data is gleaned from USGS Earth Explorer portal in order to prepare land use land cover map from 1973 to 2015.
Information about satellite data is shown in table 3.1.1. Topographical maps with a scale of $1: 50000$ namely $73 \mathrm{M} / 1,73 \mathrm{M} / 2,73 \mathrm{M} / 5,73 \mathrm{M} / 6,73 \mathrm{I} / 13$ and $73 \mathrm{I} / 14$ from Survey of India is applied to build the base layer of these satellite data.

Table 3.1.1: Details of remote sensing satellite data, Raniganj coalfield

\begin{tabular}{|cccccc|}
\hline Year & Date of Acquisition & Path/Row & $\begin{array}{c}\text { Spatial } \\
\text { Resolution }\end{array}$ & Description & Projection \\
1973 & $18^{\text {th }} \& 20^{\text {th }}$ March & $149 / 44 \& 150 / 43$ & $60 \mathrm{~m}$ & Landsat MSS & World \\
1992 & $15^{\text {th }}$ March & $139 / 44$ & $30 \mathrm{~m}$ & Landsat 5 (TM) & Geological \\
2002 & $19^{\text {th }}$ March & $139 / 44$ & $30 \mathrm{~m}$ & Landsat 7 (TM) & Survey 84/ \\
2010 & $25^{\text {th }}$ March & $139 / 44$ & $30 \mathrm{~m}$ & Landsat 7 (TM) & UTM, Zone \\
2015 & $15^{\text {th }}$ March & $139 / 44$ & $30 \mathrm{~m}$ & Landsat 8 (ETM+) & 45 \\
\hline
\end{tabular}

\subsection{Data Processing}

Data processing tasks are done from spatial and spectral enhancement menu of image interpreter tab in ERDAS Imagine software. In order to extract the entire research area for the year 1973 image stretching is performed using mosaic tool from data 


\section{Available online at www.ijrat.org}

preparation tab (Sarkar, A., 2017). Image enhancement techniques like histogram equalization, contrast stretching and tail trimming are accomplished in order to improve the visual interpretability of remotely sensed image. RGB to IHS and the reverse IHS to RGB colour space transformation functions are also accomplished for the year 2002 and 2010 to extract more information.

\subsection{Classification Scheme}

Beyond of visual interpretation signatures are selected by using AOI tool followed by parametric statistical method from the signature editor menu bar with region growing properties in Erdas Imagine. Signatures are collected from multiple areas throughout the image for a single class and are merged which belong to the same class and renamed after a land use land cover class (Lillesand et al., 2004). In this manner ten distinctive land use land cover classes are captured. These are- forest, agricultural land, fallow land, river, river sand, water body, exposure, mining lagoon, urban and excavated. On average 125 forest signatures, 153 agriculture
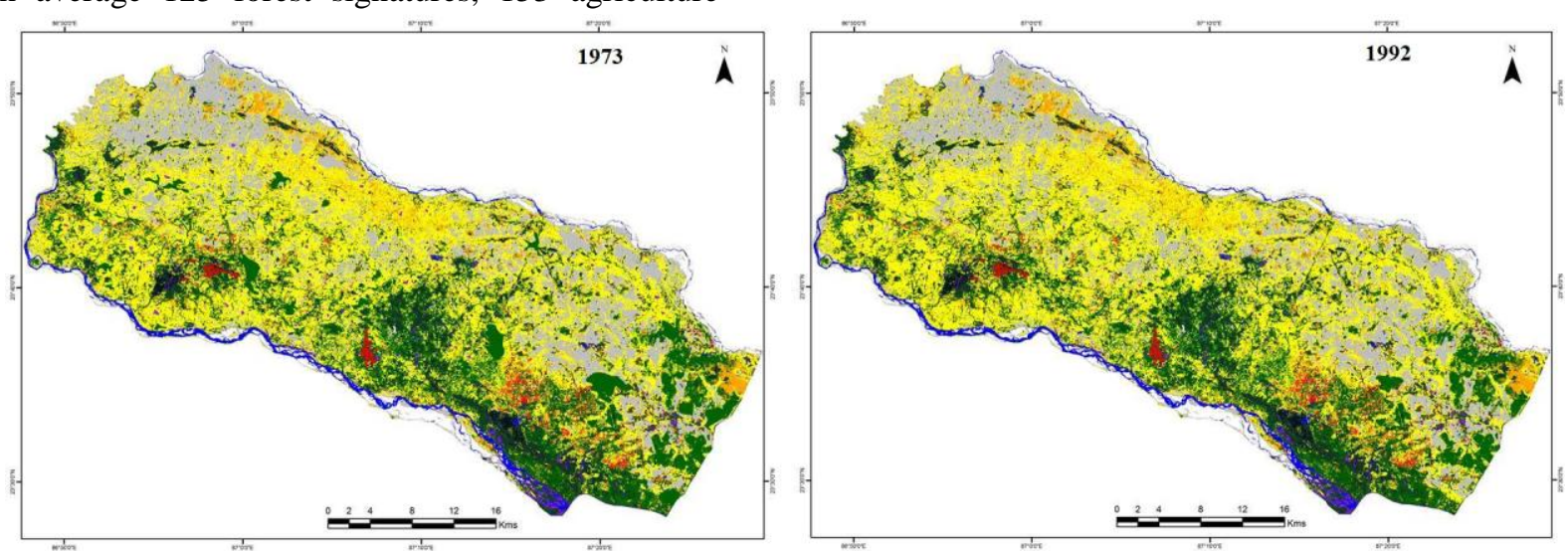

Figure 3.2.1: Landscapes map of 1973 and 1992 (clock wise), Raniganj coalfield signatures, 165 fallow signatures, 60 river signatures, 120 river sand signatures, 75 water body signatures, 87 exposure signatures, 50 lagoon signatures, 155 urban signatures and 80 excavated signatures are determined for one temporal image. Signature alarm and contingency matrix utility is also used to evaluate signatures that have been created from AOI in the image. After all these evaluations, supervised classification is performed with a distance file from classification tab/signature editor menu bar/classify/supervised to perform a supervised classification. Under parametric decision rule, maximum likelihood is selected. Then ok is clicked in the supervised classification dialog to classify the image (Sapena, M. \& Ruiz, L.A. 2015). Post classification filtering is applied from the viewer menu bar/select raster/filtering/statistical filtering (median filter) to remove unwanted discrete pixels from the thematic image and to producing homogeneous region permanently. The classified maps for 1973, 1992, 2002, 2010 and 2015 are shown in figure 3.2.1, 3.2.2 and 3.2.3 respectively 


\section{Available online at www.ijrat.org}
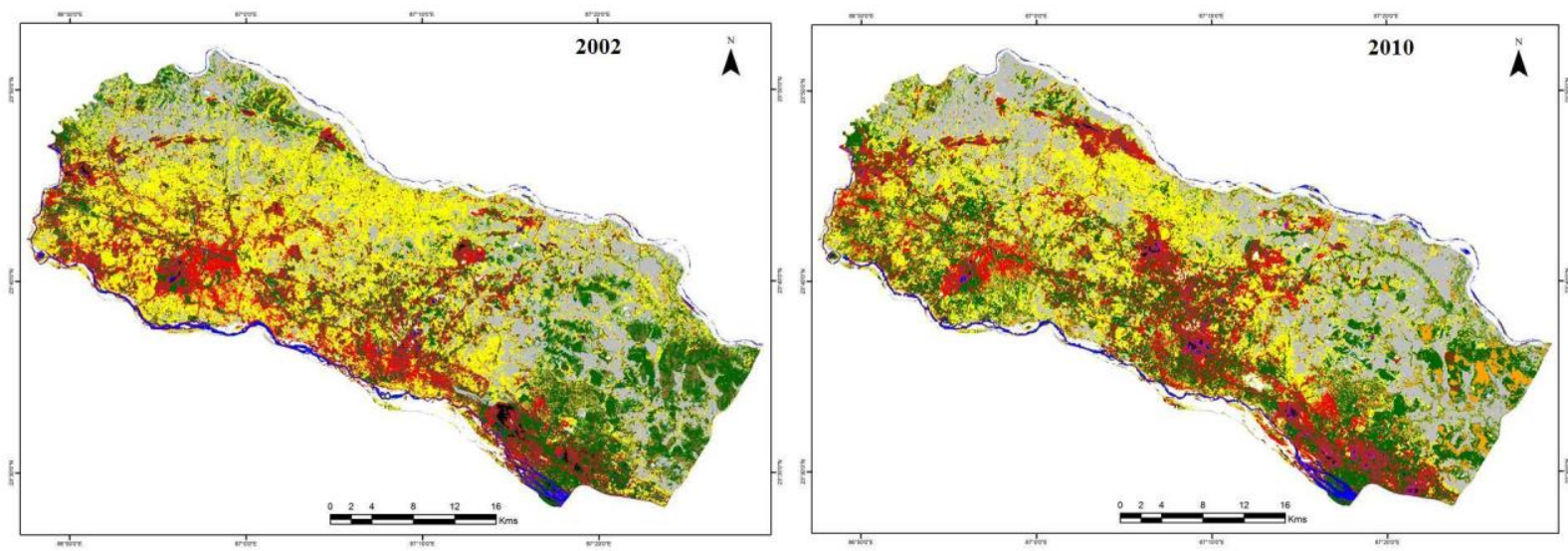

Figure 3.2.2: Landscapes map of 2002 and 2010 (clock wise), Raniganj coalfield

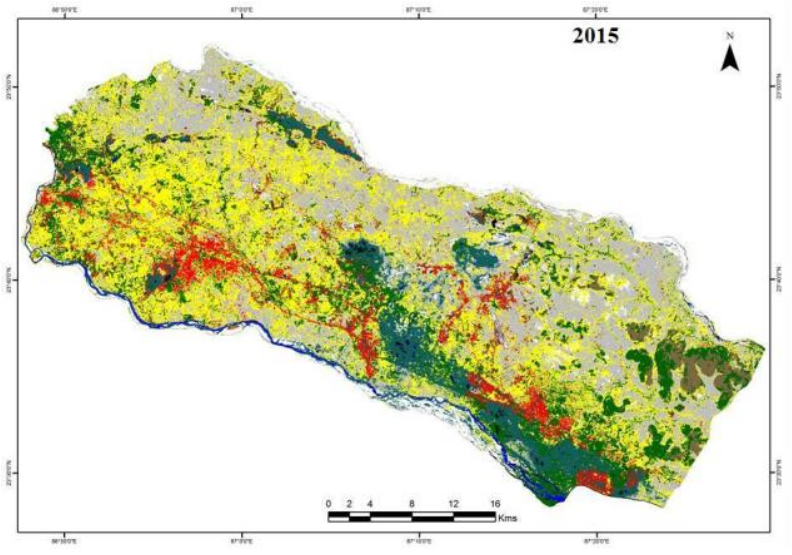

Legend

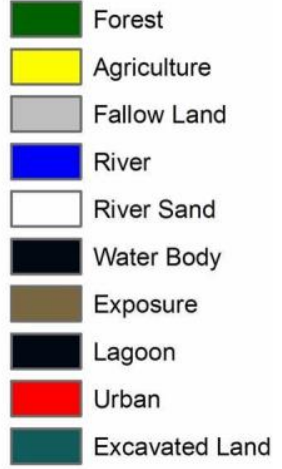

Figure 3.2.3: Landscapes map of 2015, Raniganj coalfield

3

.3 Ground Truth and Training Data Map and Photographs

With the help of Google Earth and GPS device (Garmin 72H) 950 ground truth data has been gathered for full and accurate characterization of ground truth geographic coordinates, land use and land cover attributes, species information of different landscapes using stratified random sampling method (Steven, 1987). 950 in-situ GPS waypoints are plotted in the study region according to its geographical coordinate value shown in figure 3.3.1. Photographs of different landscapes are collected and linked to the classified map according to its geographical coordinate system with the help of Google Earth in an aim to verify the classified land use land cover map with the actual surface features (Sarkar, A., 2017). Forest and excavated quarry land photograph is taken from Purusattampur area $\left(23^{\circ} 42^{\prime} 10^{\prime \prime} \mathrm{N}\right.$ and $\left.87^{\circ} 16^{\prime} 16^{\prime \prime E}\right)$ and Sonepur Bazari area $\left(23^{\circ} 39^{\prime} 40^{\prime \prime} \mathrm{N}\right.$ and $\left.87^{\circ} 11^{\prime} 0^{\prime \prime} \mathrm{E}\right)$ respectively shown in Figure 3.3.2. Photograph of agriculture and urban is taken from Kandra area $\left(23^{\circ} 25^{\prime} 42^{\prime \prime} \mathrm{N}\right.$ and $\left.86^{\circ} 42^{\prime} 41^{\prime \prime E}\right)$ and near Barakar railway station and bus stand area $\left(23^{\circ} 54^{\prime} 22^{\prime \prime} \mathrm{N}\right.$ and $\left.87^{\circ} 03^{\prime} 34^{\prime \prime E}\right)$ respectively shown in figure 3.3.3. Fallow and river sand photograph is taken from Mithali area $\left(23^{\circ} 43^{\prime} 00^{\prime \prime} \mathrm{N}\right.$ and $\left.87^{\circ} 03^{\prime} 00^{\prime \prime} \mathrm{E}\right)$ and river bed of Ajay $\left(23^{\circ} 32^{\prime} 25^{\prime \prime} \mathrm{N}\right.$ and $86^{\circ} 20^{\prime} 15^{\prime \prime} \mathrm{E}$ ) respectively shown in figure 3.3.4. Photograph of exposure land and water body is captured from Sonepur Bazari area $\left(23^{\circ} 38^{\prime} 26^{\prime \prime} \mathrm{N}\right.$ and $\left.87^{\circ} 12^{\prime} 12^{\prime \prime} \mathrm{E}\right)$ and Dalurband area $\left(23^{\circ} 42^{\prime} 36^{\prime \prime} \mathrm{N}\right.$ and $\left.87^{\circ} 01^{\prime} 52^{\prime \prime} \mathrm{E}\right)$ respectively shown in figure 3.3.5. Photograph of river and mining lagoon is captured from Barakar river $\left(23^{\circ} 59^{\prime} 12^{\prime \prime} \mathrm{N}\right.$ and $\left.87^{\circ} 04^{\prime} 10^{\prime \prime} \mathrm{E}\right)$ and Poidih abandoned mine ( $23^{\circ} 29^{\prime} 30^{\prime \prime} \mathrm{N}$ and $\left.87^{\circ} 12^{\prime} 25^{\prime \prime} \mathrm{E}\right)$ respectively shown in figure 3.3.6. 
International Journal of Research in Advent Technology, Vol.7, No.1, January 2019 E-ISSN: 2321-9637

Available online at www.ijrat.org

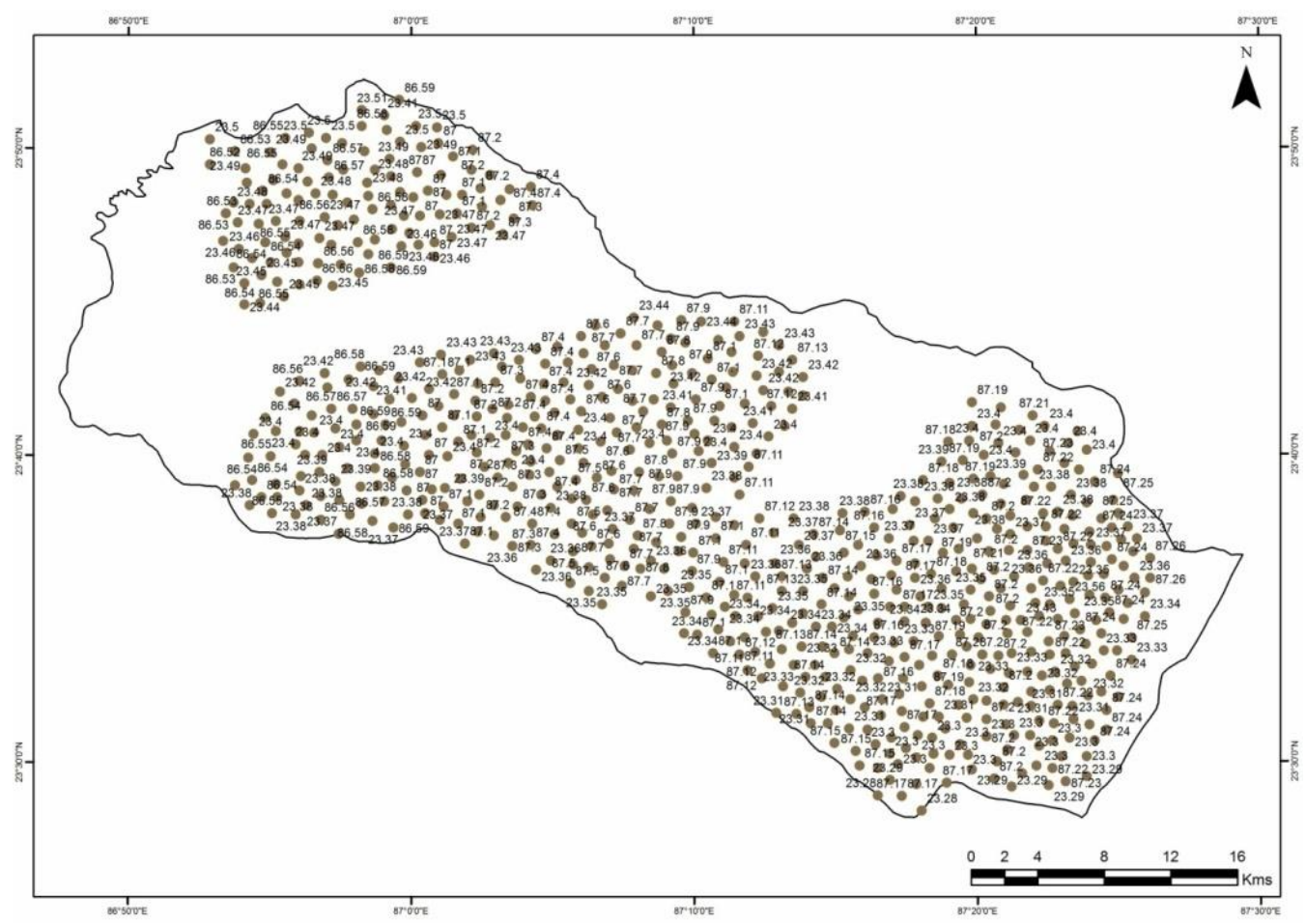

Figure 3.3.1: Ground truth verification waypoints map, Raniganj coalfield

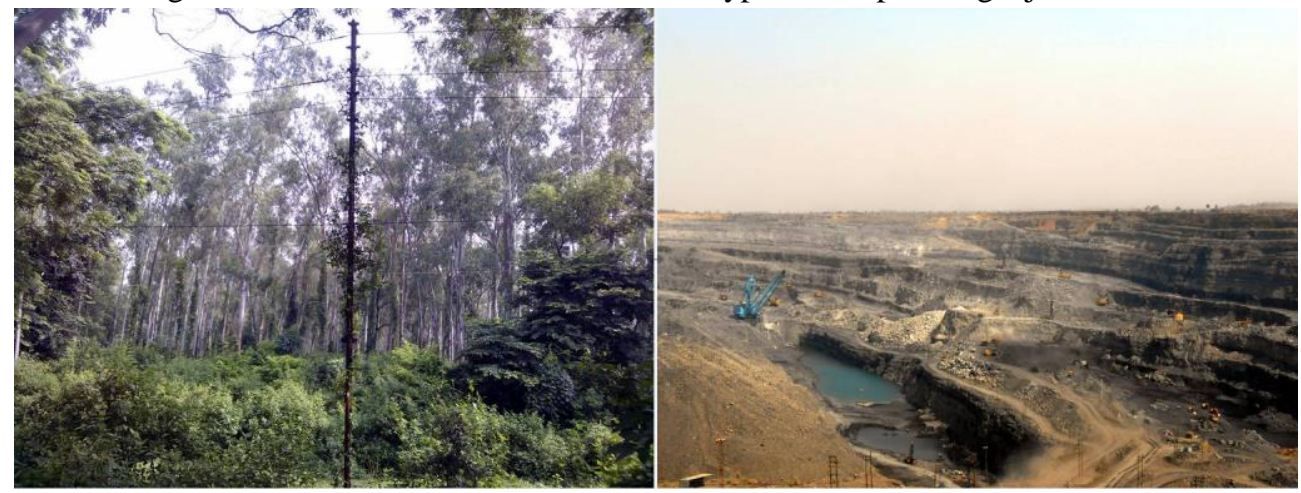

Figure 3.3.2: Landscape photograph of forest and quarry (clock wise), Raniganj coalfield

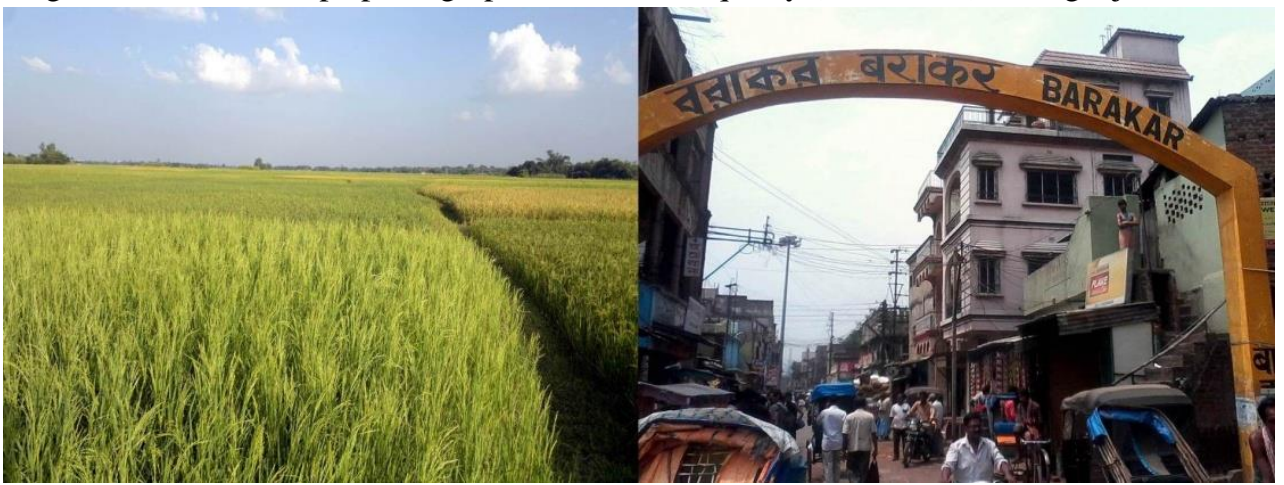

Figure 3.3.3: Landscape photograph of agriculture and urban (clock wise), Raniganj coalfield 


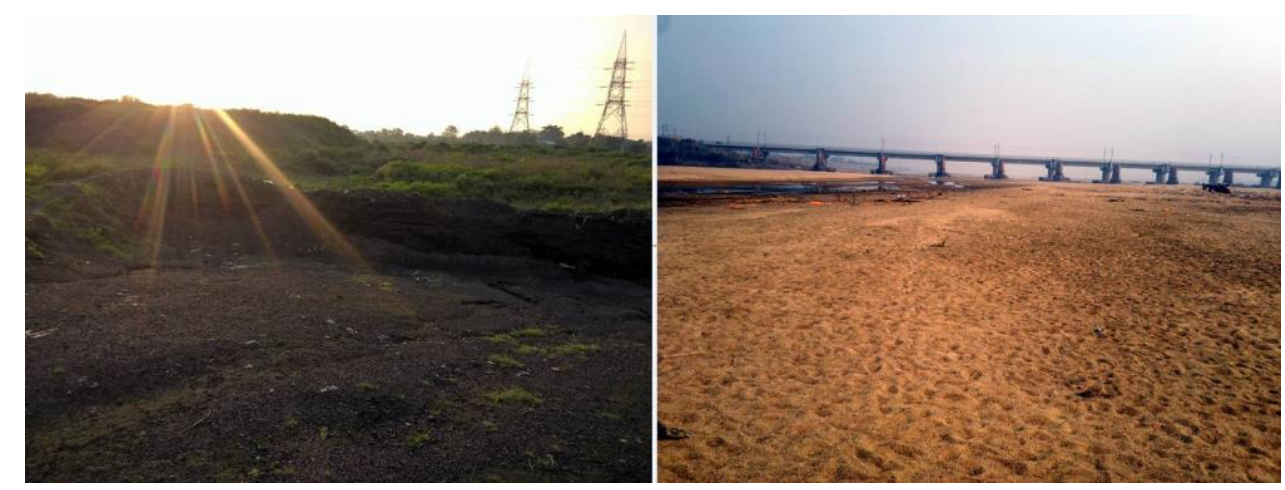

Figure 3.3.4: Landscape photograph of fallow and river sand (clock wise), Raniganj coalfield

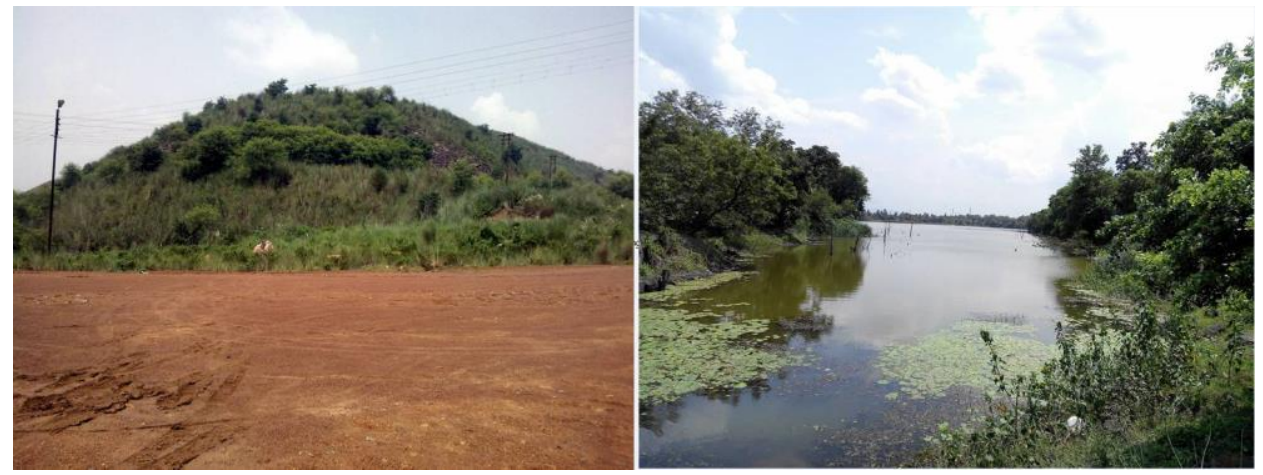

Figure 3.3.5: Landscape photograph of exposure and water body (clock wise), Raniganj coalfield

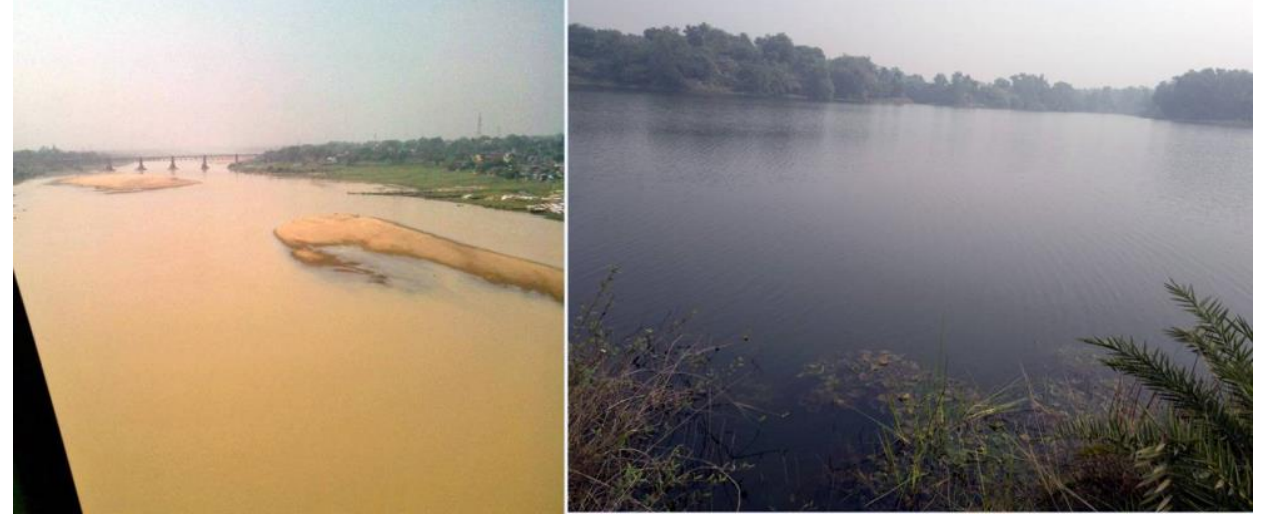

Figure 3.3.6: Landscape photograph of river and mining lagoon (clock wise), Raniganj coalfield

\subsection{Accuracy Assessment}

This work is done in ERDAS Imagine software followed by classification tab and accuracy assessment tool. The accuracy assessment algorithms are shown in table 3.4.1. Firstly stratified random sampling method is used to furnish the 878 ground truth reference data. These ground truth points are overlain on the land use land cover map and value is extracted (Kuemmerle, et al., 2006). After that a confusion matrix is generated and placed such that class membership determined by ground truth values are along the $\mathrm{x}$-axis, and class membership determined by image classification is along the y-axis (Green, 1999). When placed this way, correct values fall along the major diagonal of the matrix (Doktoringenieur, G., Buchroithner, M., Dresden, T.U. \& Prof, K. 2010). Incorrectly classified values lie in the off diagonal areas of the matrix; such that it is apparent which class they are confused with (Neill, R.V.O., Krummel, J.R., Gardner, R.H., Sugihara, G., 


\section{Available online at www.ijrat.org}

et al. 1988). Field data for validation is not available for 1973, 1992, 2002 and 2010. So it is assumed that a similar accuracy is achieved using the same methods from the 2015 land use land over map
(Green et al., 1994). Accuracy result for the land use land cover map of 1973, 1992, 2002, 2010 and 2015 is shown in table 3.4.2, 3.4.3, 3.4.4, 3.4.5 and 3.4.6 respectively.

Table 3.4.1: Algorithms for accuracy assessment, Raniganj coalfield

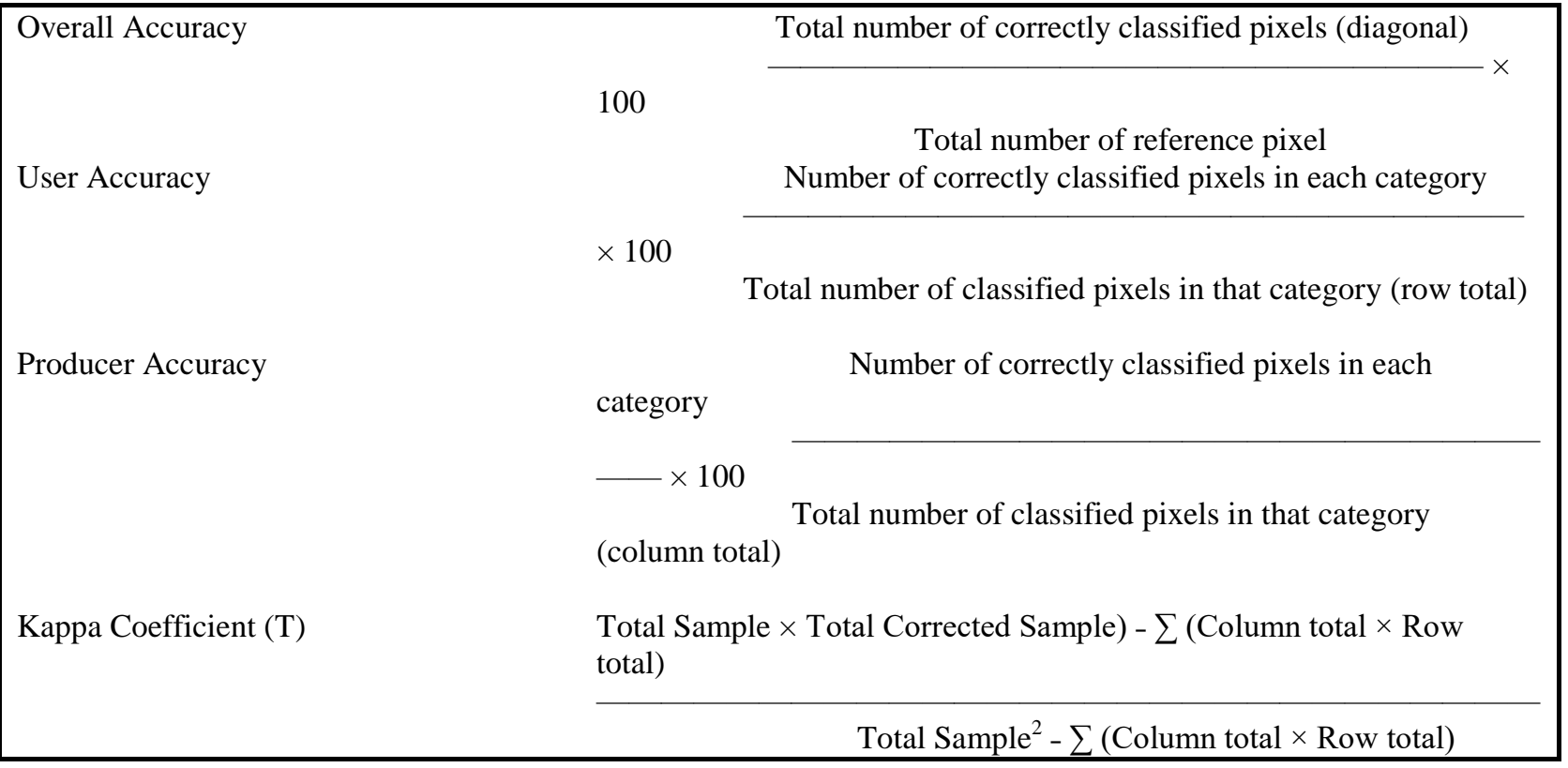

Table 3.4.2: Confusion matrix for accuracy assessment of LULC map 1973, Raniganj coalfield

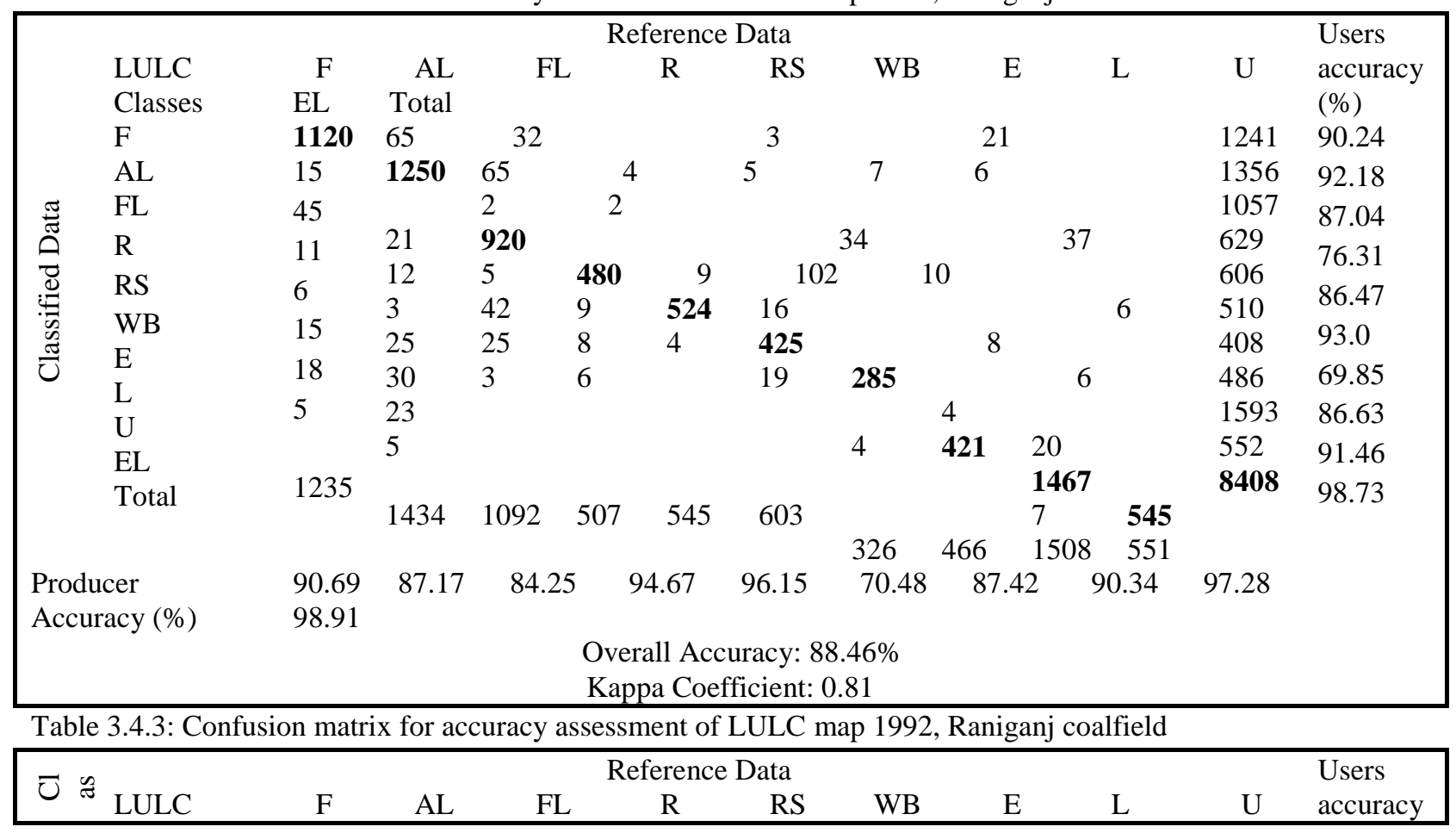


International Journal of Research in Advent Technology, Vol.7, No.1, January 2019 E-ISSN: 2321-9637

Available online at www.ijrat.org

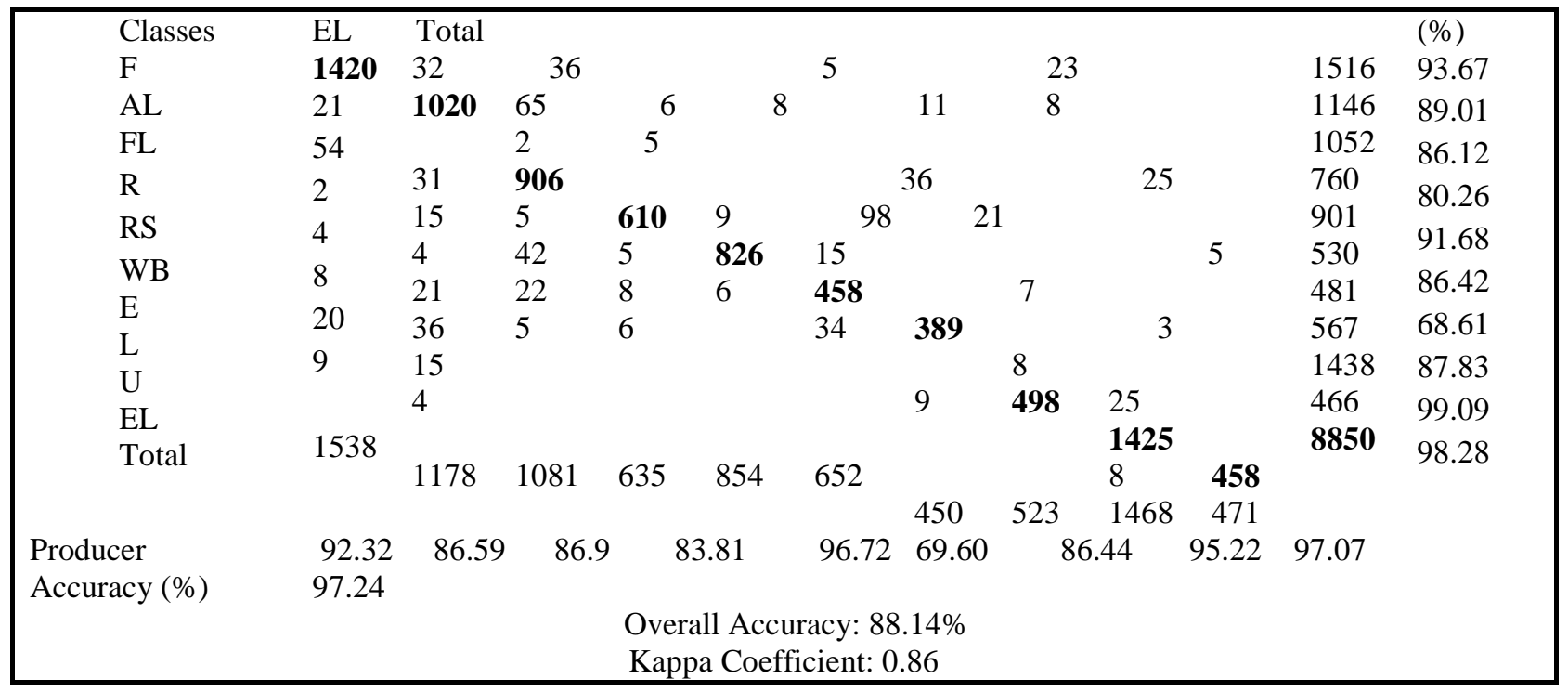

Table 3.4.4: Confusion matrix for accuracy assessment of LULC map 2002, Raniganj coalfield

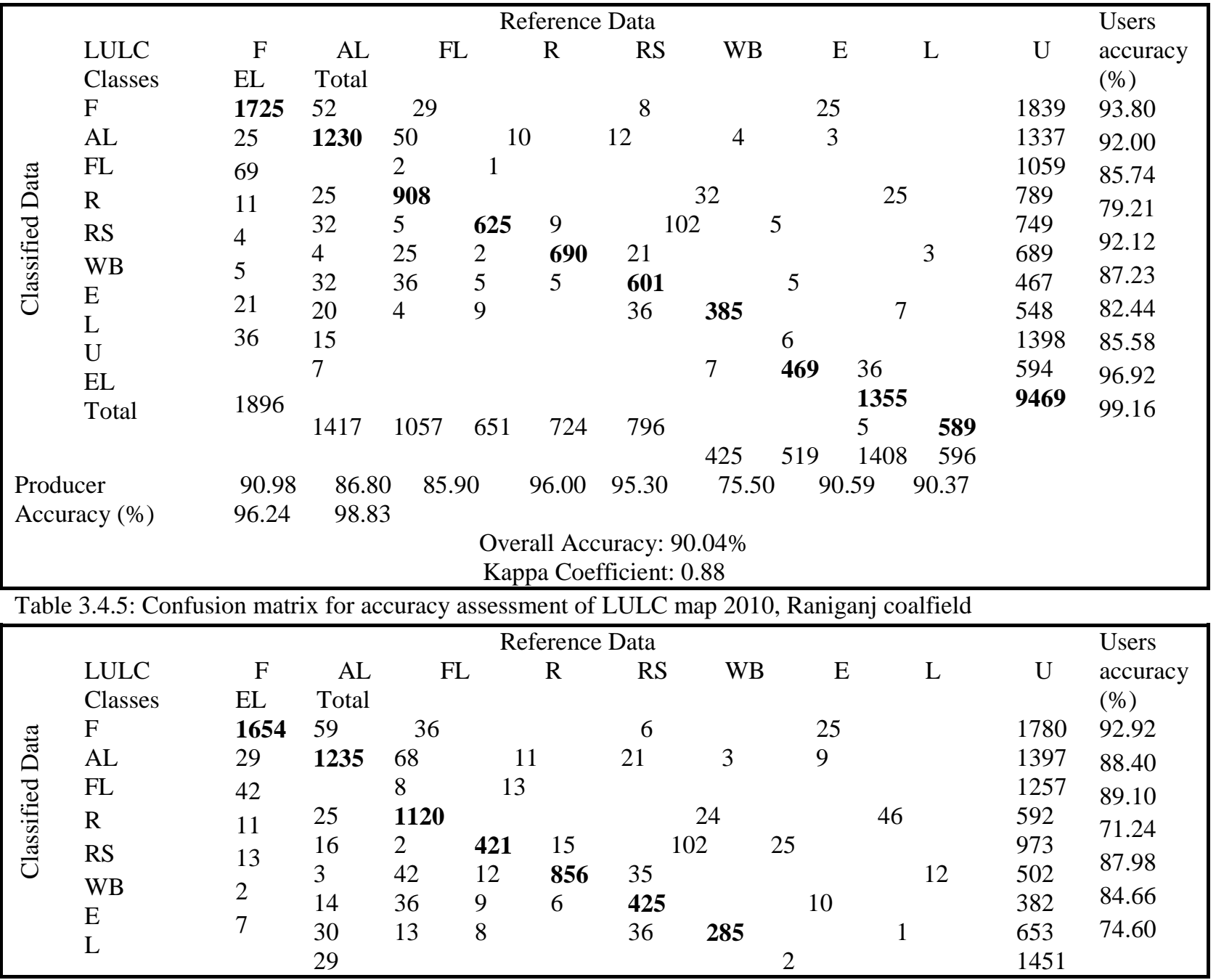


International Journal of Research in Advent Technology, Vol.7, No.1, January 2019 E-ISSN: 2321-9637

Available online at www.ijrat.org

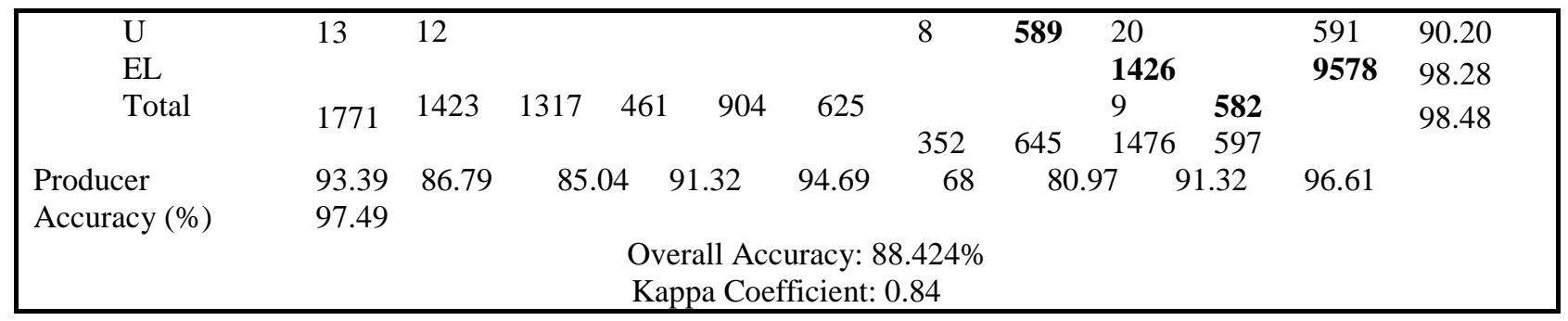

Table 3.4.6: Confusion matrix for accuracy assessment of LULC map 2015, Raniganj coalfield

\begin{tabular}{|c|c|c|c|c|c|c|c|c|c|c|c|c|c|}
\hline & LULC & $\mathrm{F}$ & AL & & & erence & $\begin{array}{l}\text { Data } \\
\text { RS }\end{array}$ & $\mathrm{W}$ & & E & $\mathrm{L}$ & $\mathrm{U}$ & $\begin{array}{l}\text { Users } \\
\text { accuracy }\end{array}$ \\
\hline & $\begin{array}{l}\text { LULC } \\
\text { Classes }\end{array}$ & EL & Total & & & & & & & & & & $(\%)$ \\
\hline & $\mathrm{F}$ & 1612 & 48 & 21 & & & 4 & & & 17 & & 1702 & 94.7 \\
\hline & AL & 20 & 1180 & 57 & 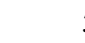 & & 7 & 8 & 3 & & & 1290 & 91.5 \\
\hline$\cong$ & FL & 56 & & 5 & 3 & & & & & & & 1146 & 88.0 \\
\hline 0 & $\mathrm{R}$ & 9 & 19 & 1008 & & & & 29 & & 34 & & 695 & 75.3 \\
\hline $\mathbb{0}$ & RS & 5 & 20 & 3 & 523 & 9 & 12 & & 10 & & 2 & 740 & 96.8 \\
\hline $\begin{array}{l}\bar{n} \\
0\end{array}$ & WB & 9 & $\begin{array}{l}2 \\
17\end{array}$ & $\begin{array}{l}36 \\
28\end{array}$ & $\begin{array}{l}8 \\
2\end{array}$ & 716 & $\begin{array}{l}12 \\
506\end{array}$ & & 4 & & & 544 & 93.0 \\
\hline$\tilde{U}$ & E & 13 & 35 & 7 & 4 & & 23 & 315 & & 2 & & 408 & 77.2 \\
\hline & $\mathrm{L}$ & 8 & 25 & & & & & & 5 & & & 466 & 90.8 \\
\hline & EL & & 8 & & & & & 6 & 423 & 20 & & 1593 & 95.5 \\
\hline & Total & 1732 & 1354 & 1160 & 517 & 734 & 677 & & & 1522 & & 702 & 97.2 \\
\hline & & & & & & & & 355 & 437 & 1603 & 692 & 9286 & \\
\hline Pro & & 93.1 & 87.1 & & 86.9 & 96.5 & 97.5 & & 4.7 & 88.7 & 96.8 & & \\
\hline & $\operatorname{acy}(\%)$ & 94.9 & 98.6 & & & & & & & & & & \\
\hline & & & & & & $\begin{array}{l}\text { all Acc } \\
\text { a Coe }\end{array}$ & $\begin{array}{l}\text { uracy: } 90 \\
\text { ficient: } 0\end{array}$ & $\begin{array}{l}.74 \% \\
92\end{array}$ & & & & & \\
\hline
\end{tabular}

\subsection{Landscape Fragmentation}

Entire tasks are done in Arc GIS 10.2 and Fragstats 4.2 software. Individual land use land cover map is converted from raster to polygon in Arc GIS following arc tool box/3d analyst tool/conversion/from raster algorithm. Then fragmentation is computed using class analyzing tool and landscape analyzing tool respectively from patch analyst tool following spatial statistics/ analyzed by class or landscape. In Fragstats software these work is done using grid method followed by input layer and analysis tab. Patch metrics, class metrics and landscape metrics indices are calculated. Area edge, shape, core area, contrast, aggregation and diversity are also calculated for different indices. The selected landscape metrics are shown in table 3.5.1.

Table 3.5.1: Selected landscape indices for Raniganj coalfield area

\begin{tabular}{|c|c|c|}
\hline $\begin{array}{l}\text { Landscape } \\
\text { Indices }\end{array}$ & Algorithm & Description \\
\hline $\begin{array}{l}\text { Number of } \\
\text { Patches }\end{array}$ & NumP $=n_{i}$ & $\begin{array}{l}\text { This describes number of patches in each class type and the } \\
\text { growth of particular patches in the region }(\mathrm{Ng}, 2006) \text {. }\end{array}$ \\
\hline Class Area & $\mathrm{CA}=\sum_{\mathrm{J}=1}^{\mathrm{n}} \mathrm{a}_{\mathrm{ij}}$ & $\begin{array}{l}\text { This is the total class area for individual land use land cover. } \\
\text { It also measures land use land cover combination (Suocheng, } \\
\text { D., 2013) }\end{array}$ \\
\hline $\begin{array}{l}\text { Percentage of } \\
\text { Land }\end{array}$ & PLAND $=\frac{\text { CA }}{\text { TA }}$ & $\begin{array}{l}\text { This is the measure of the landscape composition (Ruishan, } \\
\text { H., 2013). It describes sum of the areas of all patches divided } \\
\text { by total landscape area. }\end{array}$ \\
\hline
\end{tabular}




\section{International Journal of Research in Advent Technology, Vol.7, No.1, January 2019 E-ISSN: 2321-9637 \\ Available online at www.ijrat.org}

\begin{tabular}{|c|c|c|}
\hline $\begin{array}{l}\text { Largest Patch } \\
\text { Index }\end{array}$ & $\mathrm{LPI}=\frac{\underset{\mathrm{j}=1}{\mathrm{~m}}}{\mathrm{~A}}$ & $\begin{array}{l}\text { This is single largest patch at the class level which gives } \\
\text { indication of fragmentation, homogeneity, dominance and } \\
\text { changes within a landscape (Yu, 2006). } 100 \text { describe } \\
\text { landscape consists of a single patch while } 0 \text { denotes largest } \\
\text { patch type is absent. }\end{array}$ \\
\hline Patch Density & $\mathrm{PD}=\frac{\mathrm{n}_{\mathrm{i}}}{\mathrm{A}}(10000)(100)$ & $\begin{array}{l}\text { This is a measure of fragmentation of a patch type or } \\
\text { landscape (Mairota, 2013). higher value indicates fragmented } \\
\text { growth and vice versa. }\end{array}$ \\
\hline Total Edge Length & $\mathrm{TE}=\sum_{\mathrm{k}=1}^{\mathrm{M}} \mathrm{e}_{\mathrm{ik}}$ & $\begin{array}{l}\text { This is an absolute measure of total edge length for particular } \\
\text { patch type. Higher value indicates larger continuous patches } \\
\text { and vice versa (Lion, 2004). }\end{array}$ \\
\hline Edge Density & $\mathrm{ED}=\frac{\sum \mathrm{L}}{\mathrm{TA}} \times 10000$ & $\begin{array}{l}\text { This is the ratio of total edge distance to the total Area } \\
\text { (Poorva, 2006). Zero edge density indicates no class of the } \\
\text { landscape. }\end{array}$ \\
\hline Mean Patch Size & $\mathrm{MPS}=\frac{\mathrm{NP}}{\mathrm{TA}}$ & $\begin{array}{l}\text { This is the average size of patches of a land use class and } \\
\text { denotes landscape configuration and fragmentation } \\
\text { (Millington et al., 2003). }\end{array}$ \\
\hline $\begin{array}{l}\text { Patch Size } \\
\text { Coefficient of } \\
\text { Variance } \\
\text { Patch Size } \\
\text { Standard } \\
\text { Deviation }\end{array}$ & $\mathrm{PSCoV}=\frac{\mathrm{PSSD}}{\mathrm{MPS}}$ & $\begin{array}{l}\text { This is the coefficient of variation of patches. It measures } \\
\text { relative variability about the percentage of the mean not } \\
\text { absolute variability (Hu, 1970). } \\
\text { This is an absolute standard deviation measure of mean patch } \\
\text { areas and difference in patch size among patches (NG, 2006). }\end{array}$ \\
\hline Mean Shape Index & $\mathrm{MSI}=\sum \frac{\mathrm{PA}}{\mathrm{A}}$ & $\begin{array}{l}\text { This is a measure of average patch shape for a particular } \\
\text { patch type or for all patches in the landscape (Li et al., 2004). }\end{array}$ \\
\hline $\begin{array}{l}\text { Area Weighted } \\
\text { Mean Shape Index }\end{array}$ & AWMSI $=\frac{\text { MSI }}{\text { PA }}$ & $\begin{array}{l}\text { This is mean patch shape complexity, weighted by patch area } \\
\text { (Sadhu K., 2012). } 1 \text { AWMSI indicates all patches are circular } \\
\text { while increasing value indicates patches become complex in } \\
\text { shape. }\end{array}$ \\
\hline $\begin{array}{l}\text { Mean Perimeter } \\
\text { Area Ratio }\end{array}$ & $\mathrm{MPAR}=\frac{\sum \mathrm{PA}}{\mathrm{AR}} \times \mathrm{NumP}$ & $\begin{array}{l}\text { This is the measure of shape complexity It is the function of } \\
\text { sum of each patch perimeter, area ratio and number of } \\
\text { patches (Mairota, 2013). }\end{array}$ \\
\hline $\begin{array}{l}\text { The Shannon } \\
\text { Diversity Index }\end{array}$ & $\mathrm{SHDI}=-\sum_{\mathrm{I}=1}^{\mathrm{M}}\left(\mathrm{p}_{\mathrm{i}} \cdot \ln \mathrm{p}_{\mathrm{i}}\right)$ & $\begin{array}{l}\text { This is relative measure of patch diversity, heterogeneity and } \\
\text { fragmentation at class level of the community (Mairota et al., } \\
\text { 2013). Higher index value indicates more diverse landscape. }\end{array}$ \\
\hline $\begin{array}{l}\text { Shannon Evenness } \\
\text { Index }\end{array}$ & $\mathrm{SHEI}=-\sum_{\mathrm{i}=1}^{\mathrm{M}}\left(\mathrm{p}_{\mathrm{i}} \cdot \ln \mathrm{p}_{\mathrm{i}}\right) / 1 \mathrm{n} \mathrm{m}$ & $\begin{array}{l}\text { This is a measure of patch distribution or abundance and } \\
\text { only available at the landscape level ( } \mathrm{Li} \text { et al., 2004). } 0 \text { SEI } \\
\text { indicates low patch distribution while } 1 \text { denotes more even } \\
\text { distribution }\end{array}$ \\
\hline
\end{tabular}

13 class level indices and 2 landscape level indices for Raniganj opencast mining area are computed and displayed in table 3.5.2 and 3.5.3 respectively.

Table 3.5.2: Class level indices from 1973 to 2015, Raniganj coalfield

\begin{tabular}{|c|c|c|c|c|c|c|c|c|c|c|c|}
\hline $\begin{array}{l}\text { Landscap } \\
\text { e } \\
\text { Indices }\end{array}$ & $\begin{array}{l}\text { Yea } \\
\mathrm{r}\end{array}$ & Forest & $\begin{array}{l}\text { Agricultur } \\
\text { al } \\
\text { Land }\end{array}$ & $\begin{array}{l}\text { Fallow } \\
\text { Land }\end{array}$ & River & $\begin{array}{l}\text { River } \\
\text { Sand }\end{array}$ & $\begin{array}{l}\text { Water } \\
\text { Body }\end{array}$ & $\begin{array}{l}\text { Exposur } \\
\mathrm{e}\end{array}$ & Lagoon & Urban & $\begin{array}{l}\text { Excavat } \\
\text { ed } \\
\text { Land }\end{array}$ \\
\hline $\begin{array}{l}\text { Class } \\
\text { Area }\end{array}$ & $\begin{array}{l}197 \\
3\end{array}$ & 38143.1 & 55253.65 & $\begin{array}{l}35256.5 \\
2\end{array}$ & 4327.42 & 4165.68 & 925.82 & 3917.34 & 692.27 & 3552.24 & 7264.53 \\
\hline
\end{tabular}


International Journal of Research in Advent Technology, Vol.7, No.1, January 2019 E-ISSN: 2321-9637

Available online at www.ijrat.org

\begin{tabular}{|c|c|c|c|c|c|c|c|c|c|c|c|}
\hline & 199 & 29708.9 & 62113.8 & 32124.9 & 4018.95 & 4963.41 & 768.24 & 6723.72 & 884.7 & 8895.65 & 10764.5 \\
\hline & 200 & 27288.6 & 49115.6 & 34421.4 & 1838.07 & 8491.05 & 766.53 & 9862.74 & 1101.6 & 11219.5 & 15067.2 \\
\hline & $\begin{array}{l}201 \\
0\end{array}$ & 28193.1 & 38693.7 & 41045.9 & 961.02 & 8043.3 & 762.48 & 9575.28 & 1297.77 & 16020 & 10854 \\
\hline & $\begin{array}{l}201 \\
5\end{array}$ & 22096.8 & 44858.6 & 45843.2 & 717.75 & 8870.67 & 712.71 & 5232.68 & 1678.69 & $\begin{array}{l}19648.7 \\
2\end{array}$ & 13764.6 \\
\hline $\begin{array}{l}\text { Number } \\
\text { of }\end{array}$ & $\begin{array}{l}197 \\
3\end{array}$ & 22216 & 33061 & 13080 & 6408 & 689 & 2794 & 14388 & 1917 & 7658 & 9441 \\
\hline Patches & $\begin{array}{l}199 \\
2\end{array}$ & 41415 & 32735 & 19688 & 5952 & 821 & 2319 & 17994 & 2450 & 19178 & 13804 \\
\hline & $\begin{array}{l}200 \\
2\end{array}$ & 44170 & 31855 & 22923 & 1462 & 3454 & 2611 & 24696 & 3163 & 21226 & 17147 \\
\hline & $\begin{array}{l}201 \\
0\end{array}$ & 53172 & 36730 & 25175 & 1242 & 6043 & 3104 & 34197 & 1873 & 32263 & 26033 \\
\hline & $\begin{array}{l}201 \\
5\end{array}$ & 59981 & 37166 & 26290 & 1056 & 7868 & 3090 & 25239 & 2108 & 32603 & 17147 \\
\hline $\begin{array}{l}\text { Percenta } \\
\text { ge of }\end{array}$ & $\begin{array}{l}197 \\
3\end{array}$ & 25.12 & 36.31 & 23.64 & 3.24 & 3.63 & 1.03 & 3.34 & 0.23 & 2.68 & 5.42 \\
\hline Land & $\begin{array}{l}199 \\
2\end{array}$ & 18.47 & 38.58 & 19.97 & 2.50 & 3.08 & 0.48 & 4.18 & 0.55 & 5.52 & 6.68 \\
\hline & $\begin{array}{l}200 \\
2\end{array}$ & 17.14 & 30.86 & 21.63 & 1.15 & 5.33 & 0.48 & 6.20 & 0.69 & 7.05 & 9.47 \\
\hline & $\begin{array}{l}201 \\
0\end{array}$ & 18.13 & 24.90 & 26.41 & 0.62 & 5.17 & 0.49 & 6.15 & 0.83 & 10.31 & 6.98 \\
\hline & $\begin{array}{l}201 \\
5\end{array}$ & 13.52 & 27.45 & 28.05 & 0.44 & 5.43 & 0.44 & 3.20 & 1.03 & 12.03 & 8.42 \\
\hline $\begin{array}{l}\text { Patch } \\
\text { Density }\end{array}$ & $\begin{array}{l}197 \\
3\end{array}$ & 38.236 & 28.256 & 19.256 & 6.526 & 0.652 & 2.102 & 13.654 & 1.201 & 5.625 & 12.201 \\
\hline & $\begin{array}{l}199 \\
2\end{array}$ & 32.564 & 30.215 & 17.624 & 5.216 & 0.846 & 1.956 & 18.256 & 1.950 & 16.256 & 15.265 \\
\hline & $\begin{array}{l}200 \\
2\end{array}$ & 40.231 & 26.364 & 16.102 & 1.365 & 6.126 & 2.620 & 28.245 & 2.695 & 26.358 & 19.856 \\
\hline & $\begin{array}{l}201 \\
0\end{array}$ & 34.125 & 27.698 & 12.365 & 1.102 & 4.021 & 2.056 & 14.652 & 1.186 & 26.254 & 7.658 \\
\hline & $\begin{array}{l}201 \\
5\end{array}$ & 17.259 & 29.652 & 20.362 & 0.980 & 6.856 & 2.421 & 3.654 & 1.265 & 16.859 & 14.521 \\
\hline $\begin{array}{l}\text { Largest } \\
\text { Patch }\end{array}$ & $\begin{array}{l}197 \\
3\end{array}$ & 18.201 & 9.526 & 7.269 & 1.201 & 0.039 & 0.524 & 2.654 & 0.216 & 1.624 & 2.126 \\
\hline Index & $\begin{array}{l}199 \\
2\end{array}$ & 14.256 & 11.246 & 6.387 & 0.952 & 0.080 & 0.236 & 6.912 & 0.310 & 4.325 & 4.987 \\
\hline & $\begin{array}{l}200 \\
2\end{array}$ & 20.354 & 7.246 & 4.215 & 0.547 & 1.210 & 0.856 & 8.856 & 0.895 & 8.654 & 7.568 \\
\hline & $\begin{array}{l}201 \\
0\end{array}$ & 16.325 & 7.698 & 2.321 & 0.432 & 0.562 & 0.123 & 3.568 & 0.120 & 8.452 & 1.268 \\
\hline & $\begin{array}{l}201 \\
5\end{array}$ & 6.248 & 10.564 & 7.658 & 0.215 & 1.625 & 0.756 & 1.003 & 0.256 & 5.846 & 2.689 \\
\hline Total & 197 & 1734175 & 18226821. & 998899 & 1787515 & 583497. & 63047 & 274429 & 415808. & 136248 & 356654 \\
\hline Edge & 3 & 6.1 & 49 & 2.8 & 88 & 05 & 8 & 8.5 & 8 & 4.1 & 7.8 \\
\hline & 199 & 1350714 & 20489816. & 910173 & 1660097. & 695237. & 52316 & 471031 & 531391. & 341198 & 528487 \\
\hline & 2 & & 40 & 2.4 & 41 & 13 & 7 & 2.4 & 71 & 3.9 & 1.4 \\
\hline & 200 & 1591350 & 17846220 & 938502 & 709140 & 202992 & 63000 & 772182 & 709260 & 778008 & 814200 \\
\hline
\end{tabular}


International Journal of Research in Advent Technology, Vol.7, No.1, January 2019 E-ISSN: 2321-9637

Available online at www.ijrat.org

\begin{tabular}{|c|c|c|c|c|c|c|c|c|c|c|c|}
\hline & 2 & 0 & & 0 & & 0 & 0 & 0 & & 0 & 0 \\
\hline & 201 & 1525269 & 13695074. & 720990 & 640936.7 & 137764 & 53229 & 466769 & 417124. & 867512 & 338549 \\
\hline & 0 & 8.3 & 47 & 1.9 & 75 & 7.8 & 1 & 1.9 & 10 & 0.1 & 3.5 \\
\hline & 201 & 7968584. & 17937756. & 127741 & 383651.0 & 253339 & 58460 & 132644 & 401368. & 539948 & 491502 \\
\hline & 5 & 76 & 08 & 15 & 1 & 3.8 & 5 & 4.8 & 93 & 0.7 & 8.1 \\
\hline Edge & 197 & 55.335 & 58.168 & 31.826 & 5.696 & 1.854 & 2.000 & 8.756 & 1.322 & 4.344 & 11.382 \\
\hline Density & 3 & & & & & & & & & & \\
\hline & $\begin{array}{l}199 \\
2\end{array}$ & 43.106 & 65.391 & 29.0473 & 5.298 & 2.218 & 1.669 & 15.032 & 1.695 & 10.889 & 16.866 \\
\hline & $\begin{array}{l}200 \\
2\end{array}$ & 50.3586 & 56.474 & 29.699 & 2.244 & 6.423 & 1.993 & 24.435 & 2.244 & 24.620 & 25.765 \\
\hline & $\begin{array}{l}201 \\
0\end{array}$ & 48.267 & 43.338 & 22.815 & 2.028 & 4.3596 & 1.684 & 14.771 & 1.32 & 27.4526 & 10.713 \\
\hline & $\begin{array}{l}201 \\
5\end{array}$ & 25.115 & 56.535 & 40.261 & 1.209 & 7.984 & 1.842 & 4.180 & 1.265 & 17.017 & 15.491 \\
\hline Mean & 197 & 0.5824 & 0.5983 & 0.3709 & 1.4807 & 0.1653 & 3.0178 & 3.6729 & 2.7691 & 2.1558 & 1.2996 \\
\hline Patch & 3 & & & & & & & & & & \\
\hline Size & $\begin{array}{l}199 \\
2\end{array}$ & 1.3940 & 0.5270 & 0.6128 & 1.4809 & 0.1654 & 3.0185 & 2.6761 & 2.7693 & 2.1558 & 1.2823 \\
\hline & $\begin{array}{l}200 \\
2\end{array}$ & 1.6186 & 0.6485 & 0.6659 & 0.7953 & 0.4067 & 3.4062 & 2.5039 & 2.8712 & 1.8918 & 1.1380 \\
\hline & $\begin{array}{l}201 \\
0\end{array}$ & 1.8859 & 0.9492 & 0.6133 & 1.2923 & 0.7513 & 4.0709 & 3.5713 & 1.4432 & 2.0139 & 1.8846 \\
\hline & $\begin{array}{l}201 \\
5\end{array}$ & 2.7144 & 0.8285 & 0.5734 & 1.4712 & 0.8869 & 4.3355 & 4.8233 & 1.2557 & 1.6592 & 1.8913 \\
\hline Mean & 197 & 1.296 & 1.183 & 1.370 & 1.356 & 1.261 & 1.263 & 1.252 & 1.246 & 1.256 & 1.272 \\
\hline Shape & 3 & & & & & & & & & & \\
\hline Index & $\begin{array}{l}199 \\
2\end{array}$ & 1.323 & 1.332 & 1.342 & 1.255 & 1.415 & 1.274 & 1.281 & 1.270 & 1.242 & 1.280 \\
\hline & $\begin{array}{l}200 \\
2\end{array}$ & 1.243 & 1.346 & 1.317 & 1.367 & 1.255 & 1.221 & 1.249 & 1.229 & 1.243 & 1.281 \\
\hline & $\begin{array}{l}201 \\
0\end{array}$ & 1.317 & 1.330 & 1.344 & 1.376 & 1.307 & 1.258 & 1.297 & 1.259 & 1.289 & 1.299 \\
\hline & $\begin{array}{l}201 \\
5\end{array}$ & 1.334 & 1.362 & 1.343 & 1.317 & 1.291 & 1.211 & 1.265 & 1.21 & 1.273 & 1.267 \\
\hline Area & 197 & 8.293 & 22.181 & 10.162 & 8.226 & 2.501 & 2.603 & 1.666 & 2.558 & 0.877 & 3.792 \\
\hline Weighte & 3 & & & & & & & & & & \\
\hline $\begin{array}{l}\text { d Mean } \\
\text { Shane }\end{array}$ & $\begin{array}{l}199 \\
2\end{array}$ & 6.4555 & 28.661 & 9.256 & 7.641 & 2.982 & 2.158 & 2.856 & 3.271 & 2.197 & 5.619 \\
\hline Index & 200 & 6.214 & 26.609 & 7.368 & 7.283 & 8.480 & 1.597 & 2.046 & 2.708 & 3.653 & 5.221 \\
\hline & $\begin{array}{l}201 \\
0\end{array}$ & 5.321 & 19.609 & 10.573 & 4.404 & 6.179 & 1.646 & 2.474 & 2.016 & 3.095 & 6.654 \\
\hline & $\begin{array}{l}201 \\
5\end{array}$ & 9.052 & 24.411 & 13.652 & 3.454 & 5.099 & 1.528 & 5.874 & 1.638 & 4.155 & 9.030 \\
\hline Patch & 197 & 2363.65 & 8340.468 & 3657.92 & 3033.215 & 482.584 & 489.27 & 836.635 & 752.756 & 323.052 & 1301.80 \\
\hline Size & 3 & & & & & & 7 & & & & 4 \\
\hline Coefficie & 199 & 1841.280 & 9376.264 & 3333.11 & 2817.434 & 575.333 & 406.53 & 1436.46 & 962.866 & 809.257 & 1929.67 \\
\hline $\begin{array}{l}\text { nt of } \\
\text { Variance }\end{array}$ & 2 & 2122029 & 5178709 & $\begin{array}{l}8 \\
156006\end{array}$ & 1456741 & & 8 & 0 & 062265 & & 6 \\
\hline valialice & 2 & 2122.029 & $51 / 8.108$ & $\begin{array}{l}1509.00 \\
6\end{array}$ & 1450.141 & $\begin{array}{l}3804.18 \\
4\end{array}$ & $\begin{array}{l}213.05 \\
2\end{array}$ & $431.9 / 8$ & 902.203 & $\begin{array}{l}1195.01 \\
5\end{array}$ & $\begin{array}{l}1309.01 \\
0\end{array}$ \\
\hline & $\begin{array}{l}201 \\
0\end{array}$ & 1427.099 & 2731.143 & $\begin{array}{l}2560.32 \\
5\end{array}$ & 819.279 & 2217.86 & $\begin{array}{l}262.77 \\
4\end{array}$ & 868.819 & 453.559 & 833.837 & $\begin{array}{l}2025.09 \\
2\end{array}$ \\
\hline
\end{tabular}


International Journal of Research in Advent Technology, Vol.7, No.1, January 2019 E-ISSN: 2321-9637

Available online at www.ijrat.org

\begin{tabular}{|c|c|c|c|c|c|c|c|c|c|c|c|}
\hline & $\begin{array}{l}201 \\
5\end{array}$ & 1132.689 & 3088.358 & 3190.55 & 655.091 & 1959.53 & $\begin{array}{l}313.48 \\
0\end{array}$ & 2880.01 & 467.412 & 1228.51 & 3381.01 \\
\hline Patch & 197 & 16.690 & 140.549 & 50.484 & 19381 & 28.535 & 1.578 & 2.114 & 2629 & 0.674 & 6.748 \\
\hline Size & 3 & & & & & & & & & & \\
\hline Standard & 199 & 13.162 & 158.742 & 46.440 & 18.965 & 34.849 & 1.313 & 3.635 & 3.362 & 1.690 & 10.040 \\
\hline Deviatio & 2 & & & & & & & & & & \\
\hline $\mathrm{n}$ & $\begin{array}{l}200 \\
2\end{array}$ & 10.361 & 79.847 & 23.447 & 18.314 & 55.842 & 0.527 & 1.245 & 3.351 & 4.112 & 7.576 \\
\hline & $\begin{array}{l}201 \\
0\end{array}$ & 11.732 & 32.472 & 67.877 & 13.612 & 51.568 & 0.687 & 4.513 & 1.649 & 4.071 & 21.604 \\
\hline & $\begin{array}{l}201 \\
5\end{array}$ & 11.138 & 42.216 & 55.831 & 5.938 & 20.969 & 0.762 & 21.266 & 1.457 & 5.535 & 27.076 \\
\hline Mean & 197 & 1315.82 & 1344.857 & 1437.41 & 1273.875 & 1126.56 & 1320.1 & 1502.46 & 1389.40 & 1402.14 & 1382.71 \\
\hline Perimete & 3 & & & 0 & & 1 & 1 & 1 & 5 & 6 & 6 \\
\hline r Area & 199 & 1274.110 & 1287.594 & 1309.73 & 1183.072 & 1081.36 & 1273.5 & 1432.27 & 1359.82 & 1350.15 & 1308.82 \\
\hline Ratio & 2 & & & 3 & & 3 & 2 & 8 & 6 & 4 & 4 \\
\hline & 200 & 1151.218 & 1073.240 & 1079.85 & 1073.070 & 1116.73 & 1136.8 & 1126.80 & 1146.74 & 1143.48 & 1109.61 \\
\hline & 2 & & & 0 & & 8 & 3 & 7 & 0 & 6 & 9 \\
\hline & 201 & 1225.643 & 1183.373 & 1199.93 & 1164.910 & 1286.98 & 1257.6 & 1278.14 & 1267.19 & 1231.21 & 1146.51 \\
\hline & 0 & & & 3 & & 3 & 3 & 5 & 5 & 5 & 7 \\
\hline & 201 & 1207.298 & 1212.615 & 1215.39 & 1198.112 & 1260.59 & 1192.4 & 1330.56 & 1278.06 & 1257.13 & 1258.92 \\
\hline & 5 & & & 8 & & 7 & 7 & 4 & 0 & 9 & 8 \\
\hline
\end{tabular}

Table 3.5.3: Landscape level indices from 1973 to 2015, Raniganj coalfield

\begin{tabular}{|lll|}
\hline Year & Shannon Diversity Index & Shannon Evenness Index \\
1973 & 1.453 & 0.606 \\
1992 & 1.524 & 0.635 \\
2002 & 1.536 & 0.640 \\
2010 & 1.610 & 0.671 \\
2015 & 1.612 & 0.672 \\
\hline
\end{tabular}

\section{DISCUSSION AND FINDINGS}

Detailed statistical information of class area and percentage of land is shown in figure 4.1. Class area clearly indicates that there is a major temporal change in forest followed by agriculture and fallow. There is reduction in forest, agriculture, water body and river in 1973 to 2015. Contrary, area under fallow, river sand, exposure, lagoon, urban and excavated is increased in the abovementioned time period in northern and eastern parts of this coalfield namely Purushottompur, Sonepur and Pandaveswar area. Therefore it can be said that the part of forest cover transformed into agriculture or fallow or excavated land. Result of percentage of land indicates that agricultural land occupies highest percentage of land followed by fallow and forest. Contrary, water body occupies lowest percentage of land followed by river and lagoon. In 1973, the forest class covered an area of $25.23 \%$, but in 2015 it decreased to $13.52 \%$. The area covered by agricultural land also decreased from $36.14 \%$ in 1973 to $27.44 \%$ in 2015 . The river is decrease from $3.02 \%$ in 1973 to $0.43 \%$ in 2015 . Fallow land is increased by $10.23 \%$ in past 50 years. This shows that the area under the forest is turn in to the fallow, agriculture and urban land. Urban shows the increase by $2.32 \%$ in 1973 to $12.02 \%$ in 2015 . West and North of this coalfield Satgram, Kajora and Sripur exhibits maximum agriculture and fallow land. 


\section{International Journal of Research in Advent Technology, Vol.7, No.1, January 2019 E-ISSN: 2321-9637 \\ Available online at www.ijrat.org}
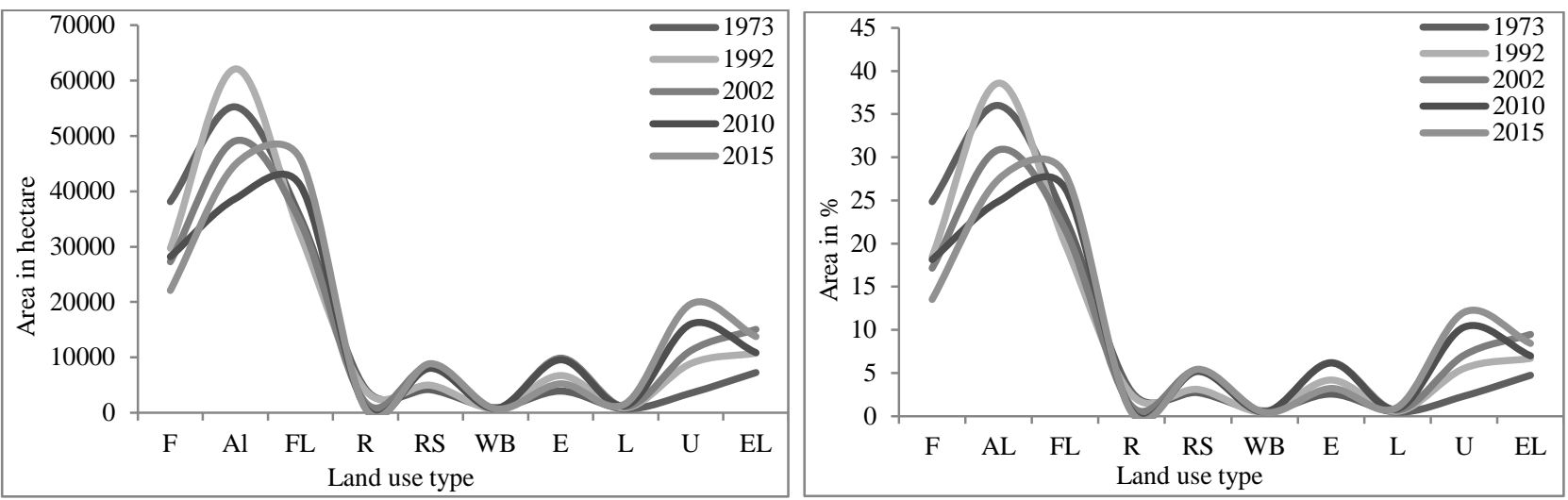

Figure 4.1: CA and PLAND 1973 to 2015 (clock wise), Raniganj Coalfield

Detailed statistical information of number of patch and patch density is shown in figure 4.2. Numbers of forest patches have increased from 22216 in 1973 to 59981 in 2015. Many forest patches isolated and converted into agriculture, fallow, quarry or urban in recent times near north-western area i.e. Sodepur, Slanpur and Pandaveswar. Number of patches for agriculture is increased from 33061 to 36730 during 1973 to 2015 is due to conversion of farmland into grassland. Number of fallow patches increased 25175 to 26290 , river sand patches increased 689 to 7868 and urban patches increased 7658 to 212226 in 1973 to 2015 respectively near Asansol and Raniganj. Agriculture patches have increased slightly because of aggregation of smaller fields into larger ones. Urban and excavated patches have increased due to growing urbanization and mining activities (Batistella, M., Robeson, S. \& Moran, E.F. 2003). Only river patches are decreased from 6408 to 1056 in 1973 to 2015 near river Ajay and Damodar. Acute increase in the number of patches indicates fragmentation. Patch density for forest, agriculture, river, exposure is significantly decreased with time. The major decreased is observed in forest 38.236 to 17.259 and river 6.526 to 0.980 since 1973 to 2015 . There is a little increase in patch density for agriculture 28.256 to 29.652 and fallow 19.256 to 20.262. Patch density drastically accelerated for river sand 0.652 to 6.856 , urban 5.626 to 16.859 and excavated land 12.201 to 14.521 . Higher patch density indicates low fragmentation within these land uses.

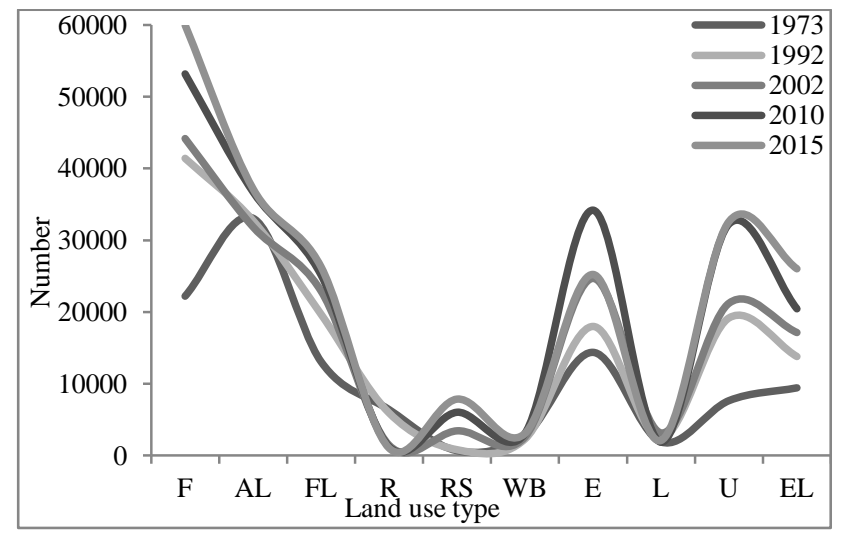

Figure 4.2: NP and PD 1973 to 2015 (c1o

Figure 4.3 shows temporal changes and detailed statistical information in total edge and edge density. There is a significant increase in total edge for fallow land, river sand, urban and excavated. Fallow

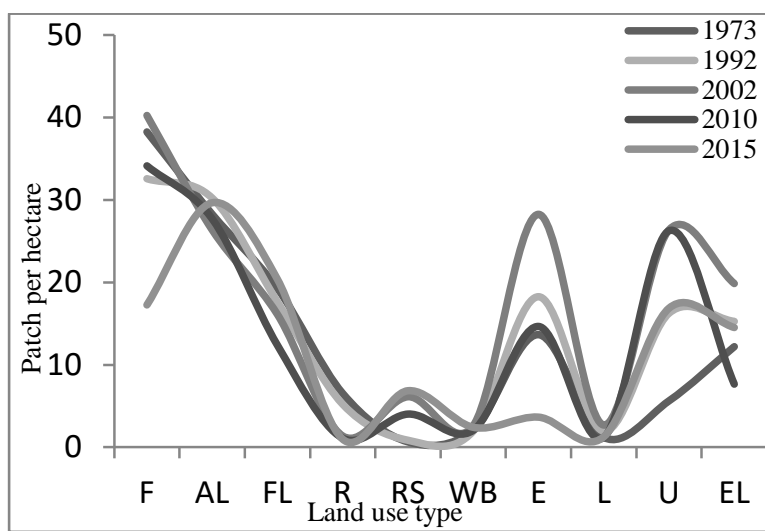

(clock wise), Raniganj Coalfield increased 9988999.8 hectare to 127774115.2 hectare where urban increased 1362484.1 hectare to 5399480.7 hectare during 1973 to 2015 near Kunstoria, Sripur, Satgram and Sodepur area. 


\section{International Journal of Research in Advent Technology, Vol.7, No.1, January 2019 E-ISSN: 2321-9637 \\ Available online at www.ijrat.org}

Agriculture, exposure and lagoon do not exhibit much difference in total edge. During the period forest, river and exposure edges have decreased drastically. Forest decreased 17341756.1 hectare to 7968584 hectare, river 1787515.8 hectare to 383651.1 hectare and exposure decreased 2744298.5 hectare to 13264448.6 hectare in 1973 to 2015 respectively.

There is an acute decrease of edge density for forest, agriculture, river, water body and exposure. Forest declined from 55.33 meter/hectare to 25.11 meter/hectare, river decreased from 5.69 meter/hectare to 1.20 meter/hectare and exposure declined from 8.75 meter/hectare to 4.18 meter/hectare during 1973 to 2015 respectively. This result denotes fragmentation in these land uses due to opencast mining and human pressure on existing land. Some land use exhibits sharp increase in edge density. Like fallow 31.82 meter/hectare to 40.26 meter/hectare, river sand 1.85 meter/hectare to 7.98 meter/hectare, urban 4.34 meter/hectare to 17.01 meter/hectare during 1973 to 2015 respectively. It is happened due to rapid urbanization process and industrial development in Raniganj coalfield area near Kulti, Asansol, Raniganj, Barakar and Disergarh.

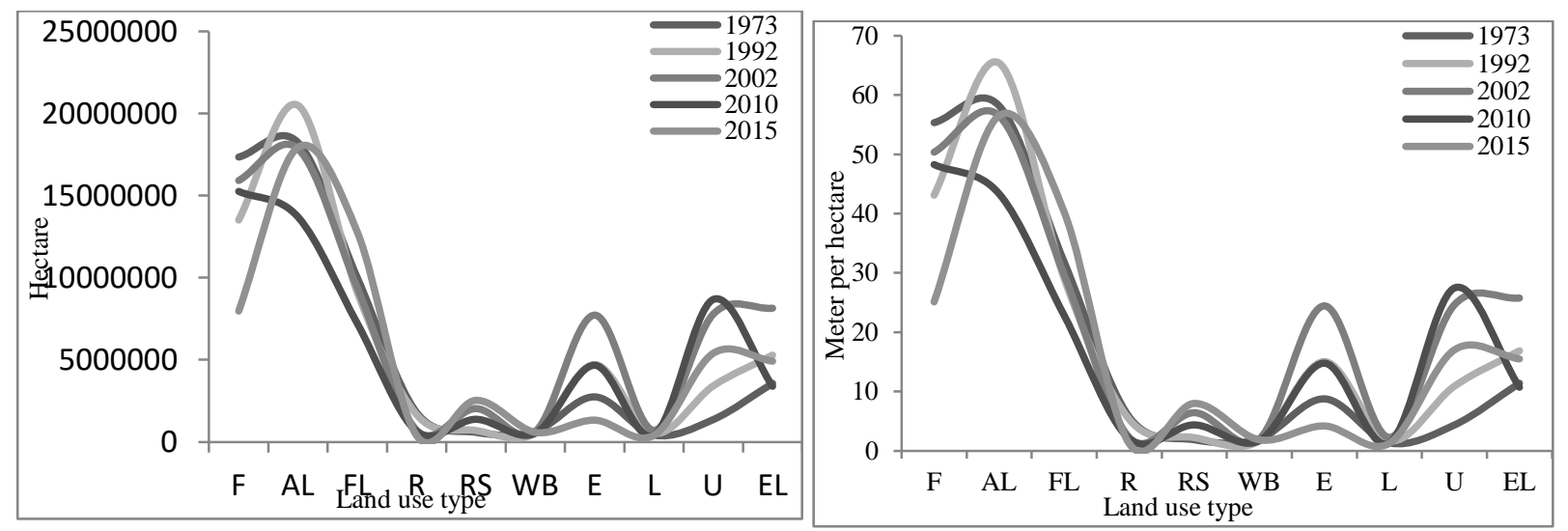

Figure 4.3: TE and ED 1973 to 2015 (clock wise), Raniganj Coalfield

Class level largest patch index and mean patch size is shown in figure 4.4. Forest is the largest patch in each class as a percentage of the total landscape. In 1973, largest patch index for forest was $18.201 \%$ of total area and then it decreased to $6.248 \%$ of total area in 2015. In 1973, the patches of forest were large and continuous. However, in 2015, the patches are scattered compared to 1992, 2002 and 2010. It is the sign of more prominent fragmentation. Urban largest patch index was $1.624 \%$ of total area in 1973 and it increased to $5.846 \%$ of total area in 2015 . It suggests that urban is increasingly becoming a dominant land use within the landscape. Urban expansion is mainly at the southern part of the study area bordering to Asansol, Durgapur, Barakar, Kulti, Raniganj city area. Largest patch index for agriculture and river sand is increased by about $1 \%$. Largest path index for fallow land is decreased from $7.269 \%$ in 1973 to $2.321 \%$ in 2010 . But it increased to $7.658 \%$ in 2015 .
In 2010 largest patch index of all land uses are decreased. Henceforth in 2015 the largest patch index for agriculture, river sand, water body, mining lagoon and excavated slightly increased and landscape becomes more complex with comparison of simple shape in 1973. Decrease in the largest patch size and increase in patches above mentioned land use land cover classes clearly indicate that patches getting clumped and tries to form a single patch therefore landscape is becoming fragmented with an acute rate. Areas covered with vegetation observed a decrease in mean patch size from 2.71 hectare to 0.51 hectare in 1973 to 2015 . Contrary urban area witnessed an acute increment from 1.65 hectare to 2.45 hectare in 1973 to 2015. Forest, agriculture, river, water body, lagoon are undergoing drastically conversion and fragmentation into quarry, fallow and urban near Jhanjra, Bankola, Kenda and Sonepur Bazari. Subsequent decrease in mean patch size in major land 


\section{Available online at www.ijrat.org}

use classes which are associated with increased fragmentation ( $\mathrm{Li}$ et al. 2004). It has been concluded that mean patch size is likely the most crucial

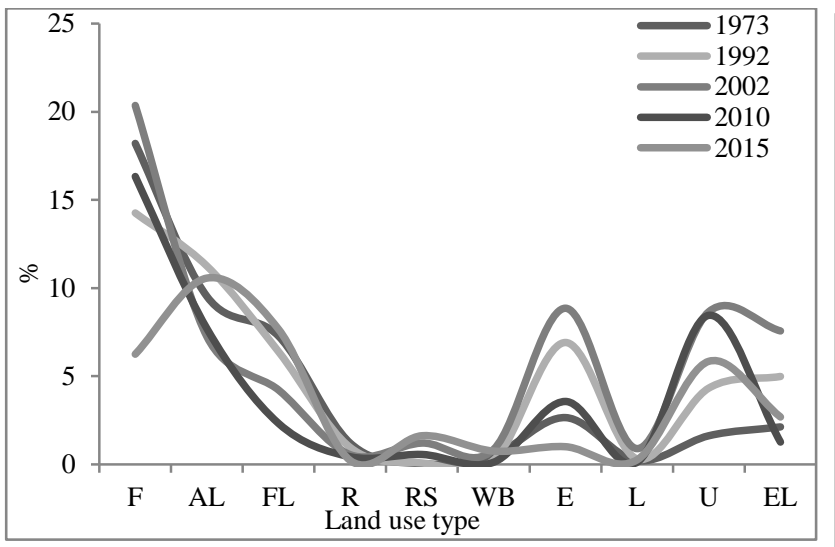

Figure indicator of fragmentation (McGarigal and Marks, 1995), therefore I suggest Fragmentation has increased in Raniganj coalfield area since 1973.

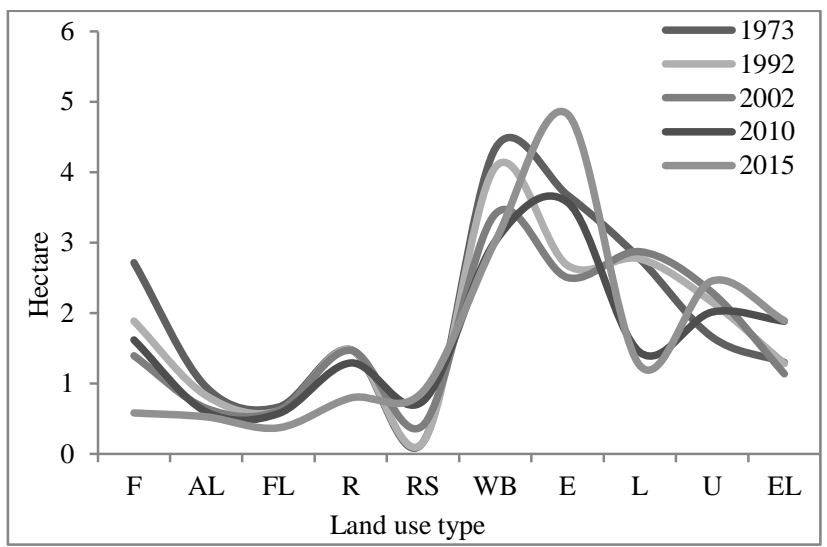

Detailed about mean shape index and area weighted mean shape index is shown in figure 4.5. Result suggests that there is little change in mean shape index for all land uses. Forest increased from 1.29 to 1.33 , agriculture 1.18 to 1.36 , river sand 1.26 to 1.29 , urban 1.25 to 1.27 in 1973 to 2015 which indicates patch shape irregularity and fragmentation. Fallow, river and mining lagoon exhibits a decrease of 1.37 to $1.34,1.35$ to 1.31 and 1.24 to 1.21 in 1973 to 2015 respectively which are less fragmented and less irregular. The area weighted mean shape index shows small change for forest from 8.29 in 1973 to 9.05 in 2015 which suggest increasing trend in shape complexity and fragmentation. The area weighted mean shape index for agriculture, fallow, river sand, excavated land and urban has increased in north and western area. Such changes indicate an expansion of these land uses around urban and coalfield areas of Raniganj coalfield. Agriculture increased from 22.18 to 24.41 and urban from 0.87 to 4.15 in 1973 to 2015 respectively. It expresses that inter patch connectivity among these land uses have decreased. This also indicates increase in shape complexity. AWMSI for mining lagoon, water body and river have declined from 2.55 to $1.63,2.60$ to 1.52 and 8.22 to 3.45 in 1973 to 2015 respectively. It indicates that these landscapes tend to be circular in shape and decrease in shape complexity.
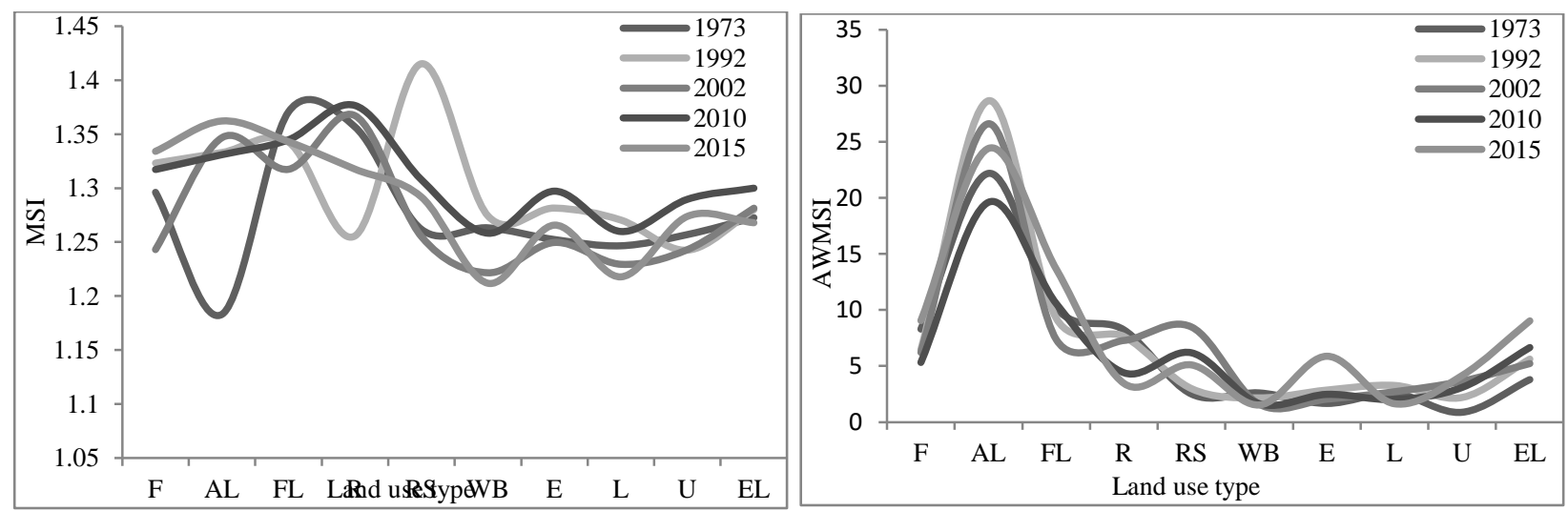

Figure 4.5: MSI and AWMSI 1973 to 2015, Raniganj coalfield 


\section{Available online at www.ijrat.org}

Figure 4.6 shows patch size standard deviation and patch size coefficient of variation. These can be misleading with regards to landscape structure in the absence of information on the number of patches or patch density (Baldi, G. \& Paruelo, J.M. 2008). Patch size standard deviation for forest, river, river sand, water body and mining lagoon decreased in tiny rate. While fallow exhibits huge decreased in PSSD from $140.54 \%$ in 1973 to $42.21 \%$ in 2015 . This indicates fragmentation growth in these landscapes. There is a huge increase of PSSD for urban and excavated land from $0.67 \%$ to $5.53 \%$ and $2.11 \%$ to $21.26 \%$ respectively in the above mentioned study period.
This means urban and excavated landscapes becoming dominant in Raniganj coalfield area. Patch size coefficient of variation is computed due to some drawback of patch size standard deviation. Patch size coefficient of variation is acutely declined for forest 2363.65 hectare to 1132.68 hectare, agriculture 8340.46 hectare to 3088.35 hectare, and river 3033.21 hectare to 655.09 hectare. Water body and mining lagoon decreased in a small rate. There is a sharp increase of PSCoV for excavated land, urban and river sand. Urban area dominantly increased from 323.05 to 1228.25 during the period.
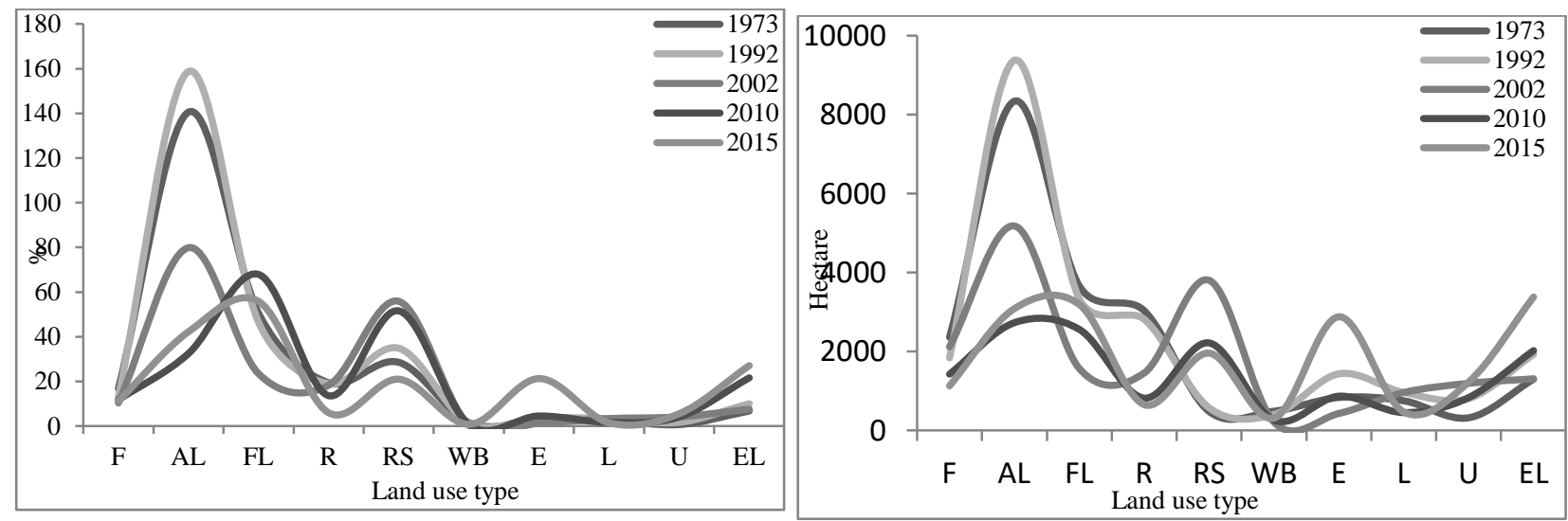

Figure 4.6: PSSD and PSCoV 1973 to 2015 (clock wise), Raniganj coalfield

Mean perimeter area ratio is shown in figure 4.7. Except of river sand, MPAR is declined for all land use categories. It indicates whole landscape has become more complicated and fragmented. Fallow exhibits acute decreased from 1437.41 hectare in 1973 to 1215.39 hectare in 2015 . There is an increase of mean perimeter area ratio for river sand from 1126.56 hectare in 1973 to 1260.59 hectare in 2017. It can be said that river sand will be increased in Raniganj coalfield area in terms of mean perimeter area ratio.

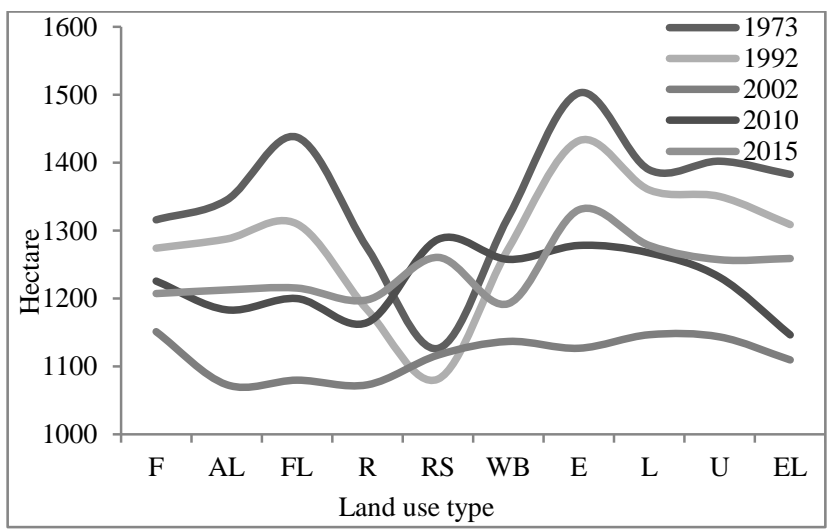

Figure 4.7: MPAR 1973 to 2015, Raniganj coalfield 


\section{International Journal of Research in Advent Technology, Vol.7, No.1, January 2019 E-ISSN: 2321-9637 \\ Available online at www.ijrat.org}

Shannon diversity index and Shannon evenness index is only computed landscape level in order to measure the fragmentation process as a whole shown in figure 4.8. Indeed SDI and SEI is likely skewed due to many small patches like water body, excavated land, exposure and mining lagoon in Raniganj coalfield area (Areendran, G., Rao, P., Raj, K., Mazumdar, S., et al. 2013). The increase in the Shannon diversity index from 1.45 in 1973 to 1.61 in 2015 indicates that landscape heterogeneity and diversity increased in Raniganj coalfield. It also means proportional distribution of different patch in the landscape therefore landscape becomes evenly distributed and fragmented. Shannon evenness index increased from 0.60 in 1973 to 0.67 in 2015. The increase in the SEI indicates that the distribution of landscape patches is more even in 2015 than it was in 1973. Outcome of these two indices clearly suggest pattern of spatial change in successive years. If these trends will carry on therefore landscape of Raniganj and adjoining become more heterogeneous and scattered.
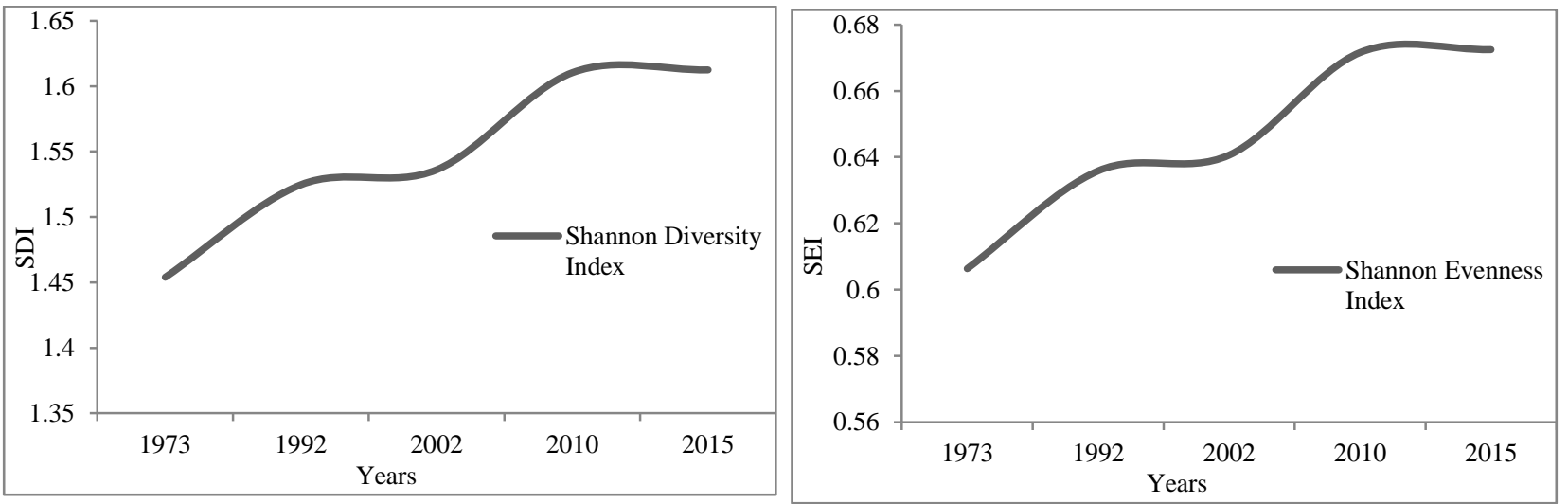

Figure 4.8: SDI and SEI 1973 to 2015 (clock wise), Raniganj coalfield

\section{CONCLUSION}

In recent time opencast mining and associated development activities are recognized as the most significant factor in order to widespread fragmentation of the landscape in Raniganj coalfield area. After assessing above mentioned results it is clear that forest patches have converted into numerous small patches and isolated in recent times. Contrary urban, fallow and excavated land will be dominated because of their increasing patch size. This huge level of land use fragmentation in Raniganj coalfield area is occurred due to extension of mining areas, development of infrastructure and residential complexes of mining industry and thermal power plants (Ampofo, S., Sackey, I. \& Ampadu, B. 2016). This intensive analysis indicates that forest, water body and river are acutely fragmented land use. These land uses are more affected in past 40 years because of declining class area, percentage of land, patch density and largest patch size. Forest and river are most prone to future fragmentation. Contrary fallow, agriculture, river sand is moderately fragmented land use because of moderate patch density, largest patch index and percentage of land. Urban is acutely effective land use in Raniganj coalfield area because of inclining class area, patch density and percentage of land in past 40 years. In the conclusion it can be said that agriculture and urban will dominate this area in near future. Large scale opencast mining, agricultural expansion, land abandonment, illegal small scale mining, deforestation and rapid urbanization predominately occurred in and around the periphery of Raniganj mining area will make the landscape or ecosystem more complex and fragmented in near future (Dupin, L., Nkono, C., Burlet, C., Muhashi, F., et al. 2013).

\section{REFERENCES}

[1] Ampofo, S., Sackey, I. \& Ampadu, B. (2016) Landscape Changes and Fragmentation Analysis in a Guinea Savannah Ecosystem : Case study of Talensi and Nabdam Districts of the Upper East region, Ghana. [Online] 8 (1), 41-54. Available from: doi:10.5539/jgg.v8n1p41.

[2] Areendran, G., Rao, P., Raj, K., Mazumdar, S., et al. (2013) Land use / land cover change dynamics 
International Journal of Research in Advent Technology, Vol.7, No.1, January 2019

E-ISSN: 2321-9637

Available online at www.ijrat.org

analysis in mining areas of Singrauli district in Madhya Pradesh , India. 54 (2), 239-250.

[3] Baldi, G. \& Paruelo, J.M. (2008) Land-Use and Land Cover Dynamics in South American Temperate Grasslands. 13 (2).

[4] Batistella, M., Robeson, S. \& Moran, E.F. (2003) Settlement Design, Forest Fragmentation, and Landscape Change in Rondônia , Amazônia. 69 (7), 805-812.

[5] Brown, D.G., Duh, J. \& Drzyzga, S.A. (2000) Estimating Error in an Analysis of Forest Fragmentation Change Using North American Landscape Characterization ( NALC) Data. 117 (June 1999), 106-117.

[6] Csorba, P. \& Szabó, S. (2010) The Application of Landscape Indices in Landscape Ecology.

[7] Das, G. \& Das, R. (2016) ' Land Use / Land Cover Status relating the Coal fire of Jharia Coal Field' - An Analytical Case Study by RS-GIS Techniques. 5 (9), 1-11.

[8] Doktoringenieur, G., Buchroithner, M., Dresden, T.U. \& Prof, K. (2010) Assessing processes of long-term land cover change and modelling their effects on tropical forest biodiversity patterns - a remote sensing and GIS-based approach for three landscapes in East Africa.

[9] Dupin, L., Nkono, C., Burlet, C., Muhashi, F., et al. (2013) Land Cover Fragmentation Using Multi-Temporal Remote Sensing on Major Mine Sites in Southern Katanga ( Democratic Republic of Congo ). 2013 (June), 127-139.

[10] Haddad, N.M., Brudvig, L.A., Clobert, J., Davies, K.F., et al. (2015) Habitat fragmentation and its lasting impact on Earth's ecosystems. (March), $1-9$.

[11] G., Raman, R. \& Punia, M. (2006) Land Use Dynamics and Landscape Fragmentation in Higher Himalaya.

[12] Hochschild, V., Shen, J., Wang, H., Koch, J., et al. (n.d.) ENVIRONMENTAL LAND USE PLANNING Edited by Seth Appiah-Opoku.

[13] Huang, J., Tu, Z. \& Lin, J. (2017) Land-use dynamics and landscape pattern change in a coastal gulf region, southeast China. [Online] 4509 (February). Available from: doi:10.1080/13504500902771891.

[14] Jaybhaye, R.G., Kale, P.K. \& Joshi, P. (2016) The Relevance of Geospatial Techniques in the Assessment of Forest Fragmentation of Anjaneri Hill , Nasik District ,. [Online] 10 (4), 1-10. Available from: doi:10.9790/2402-1004010110.
[15] Kindu, M., Schneider, T., Teketay, D. \& Knoke, T. (2013) Land Use / Land Cover Change Analysis Using Object-Based Classification Approach in Munessa-Shashemene Landscape of. [Online] 2411-2435. Available from: doi:10.3390/rs5052411.

[16] Kumar, R., Nandy, S., Agarwal, R. \& Kushwaha, S.P.S. (2014) Forest cover dynamics analysis and prediction modeling using logistic regression model. Ecological Indicators. [Online] 45, 444$455 . \quad$ Available from: doi:10.1016/j.ecolind.2014.05.003.

[17] Maitima, J.M., Mugatha, S.M., Reid, R.S., Gachimbi, L.N., et al. (2009) The linkages between land use change, land degradation and biodiversity across East Africa. 3 (10), 310-325.

[18] Neill, R.V.O., Krummel, J.R., Gardner, R.H., Sugihara, G., et al. (1988) Indices of landscape pattern. 1 (3), 153-162.

[19] Pradesh, H., Ramachandra, T. V, Kumar, U. \& Joshi, N. V (2005) Landscape Dynamics in Western Himalaya - Mandhala.

[20]R.H.U. \& Suocheng, D. (2013) Land Use Dynamics and Landscape Patterns in Shanghai, Jiangsu and Zhejiang. [Online] 4 (2), 141-148. Available from: doi:10.5814/j.issn.1674764x.2013.02.006.

[21] Samanta, P., Technical, S., Manager, P. \& Consultants, T. (2015) Impact Assessment and Changes Analysis of Land Use / Land Cover Due to Open Cast Coal Mining Activity: A Case Study of Raniganj Coal Field Area. 4 (5), 17-27.

[22] Sapena, M. \& Ruiz, L.A. (2015) Analysis Of Urban Development By Means Of MultiTemporal Fragmentation Metrics From Lulc Data. [Online] XL (May), 11-15. Available from: doi:10.5194/isprsarchives-XL-7-W3-1411-2015.

[23] Srivastav, S., Roy, S.K. \& Krishnamurthy, P.S. (2016) An Analysis Of Land Use And Land Cover Dynamics And Causative Drivers In A Thickly Populated Yamuna River Basin Of India. 14 (3), 773-792.

[24] State, J., Kumar, A. \& Pandey, A.C. (2013) Evaluating Impact of Coal Mining Activity on Landuse / Landcover Using Temporal Satellite Images in South Karanpura Coalfields and. 2 (1), 183-197.

[25] Sarkar, A., (2017) Remote Sensing Approach To Define Landscape Dynamics In Raniganj Coalfield Area Through

[26] Trend Line Analysis, Vol. 08, Issue, 09, pp.5546-5555, September, 2017. 